Issued by Sandia National Laboratories, operated for the United States Department of Energy by Sandia Corporation.

NOTICE: This report was prepared as an account of work sponsored by an agency of the United States Government. Neither the United States Government, nor any agency thereof, nor any of their employees, nor any of their contractors, subcontractors, or their employees, make any warranty, express or implied, or assume any legal liability or responsibility for the accuracy, completeness, or usefulness of any information, apparatus, product, or process disclosed, or represent that its use would not infringe privately owned rights. Reference herein to any specific commercial product, process, or service by trade name, trademark, manufacturer, or otherwise, does not necessarily constitute or imply its endorsement, recommendation, or favoring by the United States Government, any agency thereof, or any of their contractors or subcontractors. The views and opinions expressed herein do not necessarily state or reflect those of the United States Government, any agency thereof, or any of their contractors.

Printed in the United States of America. This report has been reproduced directly from the best available copy.

Available to DOE and DOE contractors from

Office of Scientific and Technical Information

P.O. Box 62

Oak Ridge, TN 37831

Prices available from (703) 605-6000

Web site: http://www.ntis.gov/ordering.htm

Available to the public from

National Technical Information Service

U.S. Department of Commerce

5285 Port Royal Rd

Springfield, VA 22161

NTIS price codes

Printed copy: A03

Microfiche copy: A01

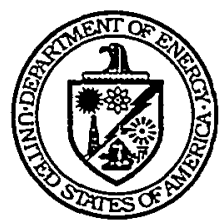




\section{DISCLAIMER}

Portions of this document may be illegible in electronic image products. Images are produced from the best available original document. 


\title{
An Assessment of Antineutrino Detection as a Tool for Monitoring Nuclear Explosions
}

\author{
Adam Bernstein and Vipin Gupta \\ Systems Research Department \\ Todd West \\ Systems Studies Department \\ Sandia National Laboratories \\ Livermore, CA 94550
}

\begin{abstract}
The antineutrino is the only real-time nuclear signature from a fission explosion that propagates great distances through air, water, and ground. The size and sensitivity of antineutrino detectors has increased dramatically in the last decade, and will continue to do so in the next, thanks in part to the renewed interest in neutrino physics brought on by the recent discovery that neutrinos may have mass. The evolution of antineutrino detectors, and the evident interest of the signature as a means for monitoring nuclear tests motivates this review of the capabilities of existing and possible future detectors as test ban verification tools.
\end{abstract}

We find that existing liquid scintillator ionization detectors, operating a few tens of meters below the Earth's surface and containing a few thousand tons of active material, could be used to monitor an area of a few square kilometers for nuclear explosions at the $1 \mathrm{kt} \mathrm{level}$. Purified water Cerenkov detectors of sizes comparable to existing detectors $\left(50,000 \mathrm{~m}^{3}\right)$ could be used to detect $1 \mathrm{kt}$ explosions at distances of a few tens of kilometers. If neutron-absorbing dopants such as sodium chloride or gadolinium could be added to purified water, the resulting background reduction would allow extension of the range for sensitivity to a pulse of 10 antineutrino events from a $1 \mathrm{kt}$ explosion out to approximately $1000 \mathrm{~km}$. Beyond $1000 \mathrm{~km}$, backgrounds from the world's nuclear reactors would become prohibitively large.

The engineering hurdles for such detectors would be formidable. The size of a doped detector operating at the $100 \mathrm{~km}$ range, suitable for cooperative monitoring of existing nuclear test sites, is about 60 times that of the largest existing water detector, and would 
require a factor of several dozen more photomultiplier tubes than what is now used in large scale physics experiments. At a price per phototube of $\$ 1000$, capital costs would amount to several billions of dollars, even for a detector at this modest range. This cost is perhaps the key obstacle to construction, along with excavation requirements and the requirement of high radiopurity for large volumes of water and dopant. Detectors sensitive to a $1 \mathrm{kt}$ explosion at a few kilometer distance would still cost tens of millions of dollars, and are unlikely to be useful except in the context of confidence-building measures. 


\section{An Assessment of Antineutrino Detection as a Tool for Monitoring Nuclear Explosions}

\section{Introduction}

Seismic, radionuclide, hydroacoustic, and infrasound detectors will all be used to verify the Comprehensive Test Ban Treaty (CTBT). Individually and in concert, these sensors will detect, identify, and locate nuclear explosions in the atmosphere, underwater, or underground. They are part of a proposed international monitoring system that, while comprehensive, has limitations. Perhaps the most significant limitation is that the system may not be able to reliably identify, in real time, a very low yield ( $<\sim 1 \mathrm{kt}$ ), underground explosion as a nuclear event, particularly if evasion methods such as seismic decoupling are employed. ${ }^{1}$ This limitation exists because the shock waves and radiation from the underground explosion are damped or absorbed by the Earth itself. The Earth is, however, transparent to one form of nuclear radiation - the antineutrino. Detection of the intense burst of antineutrinos generated by a nuclear blast might make it possible to identify such explosions even at low yield or when decoupled, thereby helping to resolve the ambiguities that exist in the current monitoring system.

The basic idea of detecting the antineutrinos emitted by nuclear explosions has been broached occasionally since the antineutrino was first discovered in the $1950 \mathrm{~s}^{2}$. Since that time, advances in detection technology have led to the construction of very large, low noise detectors, with a $50,000 \mathrm{~m}^{3}$ detector (SuperKamiokande, completed in 1995) already built in Japan, and a 1 billion $\mathrm{m}^{3}\left(1 \mathrm{~km}^{3}\right)$ detector proposed for construction at the South Pole. ${ }^{3}$ Given the recent and planned increases in scale and sensitivity of neutrino detectors, the unique properties of the neutrino signature from nuclear explosions, and the apparent absence of a scientific review of neutrino detectors' capabilities in this regard, it is useful to investigate the potential of these systems and consider the improvements that would be required to make them useful for test ban verification purposes.

In this paper, we discuss the prospects for detecting the antineutrino pulse produced by low yield fission explosions. We consider two possible applications: cooperative monitoring at modest distances from a test (less than a few $100 \mathrm{~km}$ ) and independent remote monitoring at great distances (1000 km to an Earth diameter).

We start with a brief review of some important existing verification technologies, including the four technologies officially designated for use in the international CTBT verification regime. Next, we consider possible ways in which antineutrino detectors might be useful as supplementary monitoring tools in the context of test ban verification. We then investigate the sensitivity of two types of antineutrino detectors to fission antineutrino bursts, including estimates of range, detector size, backgrounds, and infrastructure requirements. In the course of the analysis, we discuss the limits placed on the detectors' functionality by physics and engineering considerations. We conclude by assessing the potential of antineutrino detectors for test ban verification and possible avenues for future research. 


\section{Existing CTBT Verification Technologies}

A variety of on-site and remote sensor technologies can be used for verification of the Comprehensive Test Ban Treaty, with each offering unique sensing capabilities. The technologies can be used to search for nuclear explosions conducted in the atmosphere, underwater, underground, or outer space. The use of two or more sensors of different types increases the reliability of detection, and can help identify otherwise ambiguous events.

\section{On-site Technologies}

Candidate on-site nuclear detonation detection technologies include seismic, radionuclide, hydrodynamic, gamma, X-ray and neutron detectors as well as groundbased EMP sensors. One possible benefit of some of these technologies is the ability to detect an intrinsically nuclear signature from a low yield test. However, effective operation of such systems is highly intrusive, requiring in some cases that the sensors have an unobstructed view, typically within meters of any suspect activity. On-site seismic, radionuclide, and EMP sensors can operate at greater distances from any monitored activity, but the probability of detecting and identifying illicit tests is diminished. On-site seismic sensors have the additional drawback (from the host country's perspective) of being able to detect vibrations produced by nearby legal, but sensitive, activities unrelated to nuclear testing.

\section{Remote Technologies}

Remote sensing technologies, in combination, provide extensive detection capabilities, but each technology alone is essentially limited to one detonation medium. The technologies for remotely detecting and identifying nuclear explosions in the atmosphere or outer space are highly capable; several different sensor systems can be used to capture the nuclear signature of an atmospheric or exoatmospheric explosion seconds to days after the event. Five of these (Bhangmeters, and X-ray, gamma ray, neutron, and EMP detectors) are space-based and designed to see the immediate particle and electromagnetic radiation from the explosion. Radionuclide sensors can detect the longlived radioactive gases and particulates from the explosion.

In contrast, the five remote sensor technologies for detecting and identifying underwater or underground nuclear tests (radionuclide, seismic, infrasound, hydroacoustic, and

\footnotetext{
${ }^{a}$ The CTBT includes provisions for post-event On-site Inspections, which can include the use of specified sensing equipment sometimes referred to as "On-site Technologies". The use of these sensors is intended to resolve ambiguous events detected in other systems (e.g. the remote sensing systems). In this paper, we use the term "On-site Technologies" in a more generic way to refer to any sensor deployed within a short distance of a suspected test site for the purpose of registering a signal from a nuclear event. Since there are no provisions in the CTBT for monitoring with this larger class of sensors, permission to use them would have to be granted by separate bilateral agreements.
} 
satellite imaging) are not as robust. Only radionuclide sensors can see an intrinsically nuclear signature from a fission explosion, and then only if the contained explosion accidentally vents into the atmosphere. The other four technologies can see various blast effects, but not direct nuclear radiation effects. Consequently, by utilizing evasion tactics such as decoupling, deeper burial, camouflage, concealment, and deception, it may be possible to conduct low-yield nuclear explosions underground that would escape detection altogether, or be incorrectly identified as non-nuclear phenomena (e.g., earthquakes, rockbursts, and chemical explosions). ${ }^{4}$

From a verification standpoint, the incorrect classification of a seismic event is a particularly vexing problem. In the last ten years alone, there have been three documented cases where natural earthquakes were incorrectly identified as nuclear explosions. Two of these cases happened in January and August of 1996 near the Russian nuclear test site at Novaya Zemlya. In addition to the occasional occurrence of false positives, there is the more persistent problem of possible false negatives - the incorrect classification of low yield underground nuclear explosions as innocuous events. ${ }^{6}$ Although seismic sensors have the best chance of detecting a low yield underground test, the relatively weak signal $\left(m_{b} \sim 2.0-3.0\right)$ is not inherently nuclear and would be just one of the thousand estimated ambiguous seismic signals produced by earthquakes worldwide each year. ${ }^{7}$ As a result, the rare occurrence of a clandestine, underground nuclear test could be lost in the noise.

The problems of ambiguous signals in the seismic remote monitoring system, and the intrusiveness of existing on-site monitoring systems, raise the question of whether other sensors might give useful supplementary information. In the following section we consider how antineutrino detectors might fit into the existing verification framework.

\section{Applications of Antineutrino Detectors for Verification}

In many ways, the burst of antineutrinos given off by every fission explosion provides an ideal nuclear detonation signal. A large number are made in every fission explosion roughly 5-10 percent of the energy of the nuclear explosion is in the form of antineutrinos $\left(10^{24}\right.$ antineutrinos per $\mathrm{kt}$ of fission yield). These antineutrinos are produced by nuclear $\beta$ decay of fission products in approximately 10 seconds (see Figure 3 ) and then travel at the speed of light in all directions. The resulting burst of antineutrinos is an intrinsically nuclear signal - no natural terrestrial source produces an antineutrino pulse of this intensity. Since the material around the nuclear device does not appreciably affect the antineutrinos, the antineutrino signal is independent of the medium in which the explosion takes place. By contrast, medium dependent effects must be corrected for in other sensor systems in order to reconstruct the original signal. Indeed, the antineutrino signal is impossible to mask, since antineutrinos easily pass through the entire Earth without being significantly attenuated. In sum, antineutrinos provide the only known, intrinsically nuclear signal that travels large distances from underground nuclear explosions. 
Unfortunately, the same transparency that makes antineutrinos impossible to mask also makes them very difficult to detect. It is only recently that detectors large enough to be of interest for nuclear detonation detection have been seriously contemplated. ${ }^{8}$ For example, the largest current antineutrino detector, SuperKamiokande, is of the appropriate volume $\left(50,000 \mathrm{~m}^{3}\right)$ to detect a burst of five antineutrinos from a $1 \mathrm{kt}$ detonation $25 \mathrm{~km}$ away. The proposed next generation of detectors includes a design for a detector of the appropriate volume $\left(1 \mathrm{~km}^{3}\right)$ to detect antineutrinos from a $1 \mathrm{kt}$ detonation about $3000 \mathrm{~km}$ distant. In the following sections, we consider possible applications of antineutrino detectors for both local and remote monitoring of nuclear tests.

\section{Cooperative Monitoring Applications of Antineutrino Detectors}

\section{Subcritical Test Monitoring}

The CTBT bans both full scale and low yield nuclear explosions down to zero nuclear yield. The US and Russia have announced their intention to perform "subcritical" tests in which conditions for an exponentially growing fission chain reaction are not created. The US intends to conduct two to four underground subcritical tests per year at the U1a facility at the Nevada Test Site, having already begun with a test code-named Rebound, conducted in $1997 .^{9}$ The activities associated with these tests are quite difficult to distinguish from those connected with low yield nuclear tests. The problem for the US and Russia is to demonstrate that these declared subcritical tests are not actually low yield supercritical tests, and are not being used to mask such tests. At the same time, the two countries are reluctant to use highly intrusive monitoring systems that could reveal sensitive information. Since allowed activities at the test site do not typically produce antineutrinos, ${ }^{10}$ and since the antineutrino pulse is the only intrinsically nuclear signal that can be reliably detected outside of the test cavity, antineutrino detectors, if feasible, would be excellent tools for monitoring a declared sub-critical test site.

\section{Reduce Need for On-Site Inspections}

An on-site antineutrino monitoring regime would also have value in resolving ambiguous events that may appear to be nuclear explosions with yields much larger than a few kilograms or even a few tons. The CTBT provides for on-site inspections to resolve suspicious events, but these inspections are not immediate, require a political process for approval, and can be highly intrusive over a very large area (up to $1000 \mathrm{~km}^{2}$ ). A nonintrusive local monitoring regime could greatly reduce the likelihood of the host nation being required to submit to such an intrusive, and perhaps frivolous, CTBT verification inspection within the detector's area of regard. For example, a detector range of $100 \mathrm{~km}$ for a $1 \mathrm{kt}$ test is sufficient to cover most existing nuclear test sites (see Figure 1). Such a detector would greatly reduce the concern that decoupled kiloton-level nuclear detonations occurring at these test sites were missed by the world wide seismic network, and would reduce the need for intrusive inspections. As an additional benefit, a detector 
capable of sensing antineutrinos from a $1 \mathrm{kt}$ explosion $100 \mathrm{~km}$ away would be able to detect an explosion with a few kilograms of yield $100 \mathrm{~m}$ away.

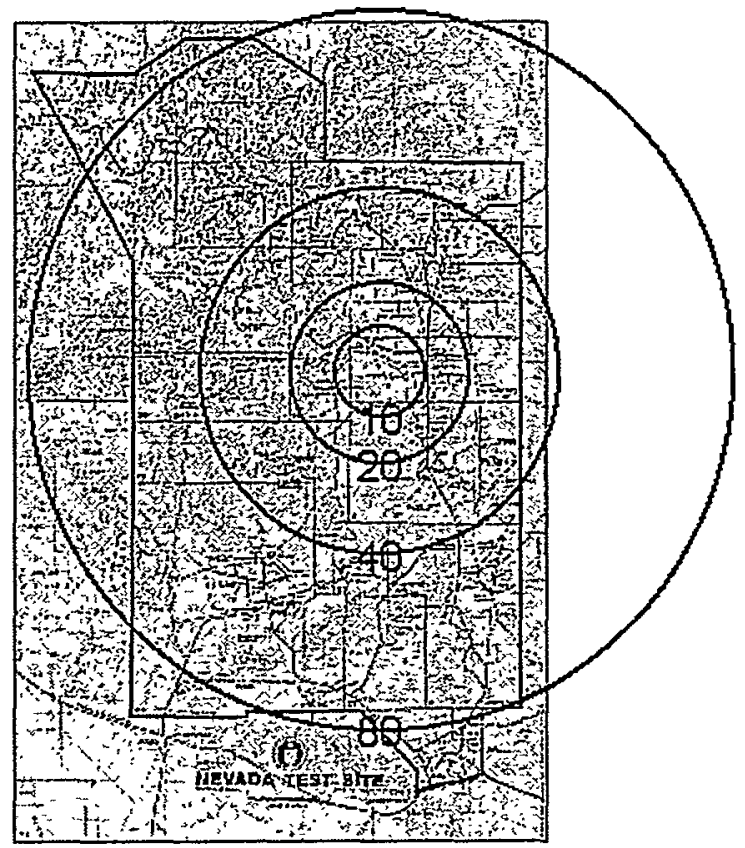

Figure 1: Nevada Test Site with range circles at $10 \mathrm{~km}, 20 \mathrm{~km}, 40 \mathrm{~km}$, and $80 \mathrm{~km}$.

In general, a local monitoring system would build confidence and reduce misunderstandings of the sort produced by the 1996 Novaya Zemlya seismic events. A local monitoring regime could be instituted under the CTBT or through separate agreements (e.g., US-Russia, India-Pakistan-China). Such agreements would build on the historical interest in on-site verification dating back to the Joint Verification Experiments associated with the Threshold Test Ban Treaty.

\section{Applications of Remote Detectors}

As hard as it is to detect antineutrinos, it is even more difficult to extract directional information from this signal. Thus, long range detectors should be viewed as a complement to other technologies that can provide more specific information on the location of possible nuclear tests. Used in this way, an antineutrino detector would indicate that a nuclear detonation had occurred at a specific time (with an uncertainty of no more than a few tens of seconds) within a certain distance of the detector, while other technologies, such as seismic detection, would provide more precise location of suspicious events that occurred in this time window. Long range antineutrino detectors, operating synergistically with detectors relying on other physical phenomena (principally seismic) would greatly reduce the problem of decoupling, decrease the number of false alarms, and increase the chances of successful and convincing attribution. Antineutrino 
detectors would also provide additional data on the yield of the nuclear device and possibly the fraction of yield from fission versus fusion.

The ideal antineutrino detector would have a range of at least the diameter of the Earth $(12,800 \mathrm{~km})$ for a $1 \mathrm{kt}$ detonation. However, shorter range detectors would have value as well. For example, a detector with a range of $3000 \mathrm{~km}$ for a $1 \mathrm{kt}$ yield, located in Greenland could detect a $1 \mathrm{kt}$ detonation at Novaya Zemlya or a detonation with a yield above, approximately, $10 \mathrm{kt}$ anywhere on Earth.

\section{Antineutrino Burst Detection Methods}

The low interaction probability of antineutrinos imposes two general requirements on all candidate detectors: either the antineutrino flux must be high, or the detector volume large. To ensure a high flux, the detector should be as near as possible to the site of the explosion, as might be possible in the context of cooperative on-site monitoring. If political and practical constraints do not allow this, the detector volume must increase. At the extreme limit of an Earth diameter $(12,800 \mathrm{~km})$, sensitivity to even five events from a $1 \mathrm{kt}$ explosion would require a detector with a volume equivalent to that of a cube $2.3 \mathrm{~km}$ on a side.

In the following sections we define the size, composition, and proximity of detectors sensitive to nuclear explosions with yields at or below the $1 \mathrm{kt}$ level. We begin by describing the properties of the fission antineutrino burst.

\section{Number, Time and Energy Distributions of Antineutrinos Produced in a Fission Explosion}

About 5-6 antineutrinos $(\bar{v})$ are produced per fission in a nuclear explosion. The total number is directly proportional to the explosive yield.

1) $\quad N_{\bar{v}} \cong 6 \cdot N_{\text {fiss }} \cdot Y$

with

$\begin{array}{ll}\mathrm{Y}(\mathrm{kt}) & \rightarrow \text { yield } \\ \mathrm{N}_{\text {fiss }}=1.45 \times 10^{23} & \rightarrow \text { number of fissions } / \mathrm{kt}\end{array}$ 
Figure 2 shows the approximate energy distribution of the antineutrinos emitted by a fission explosion.

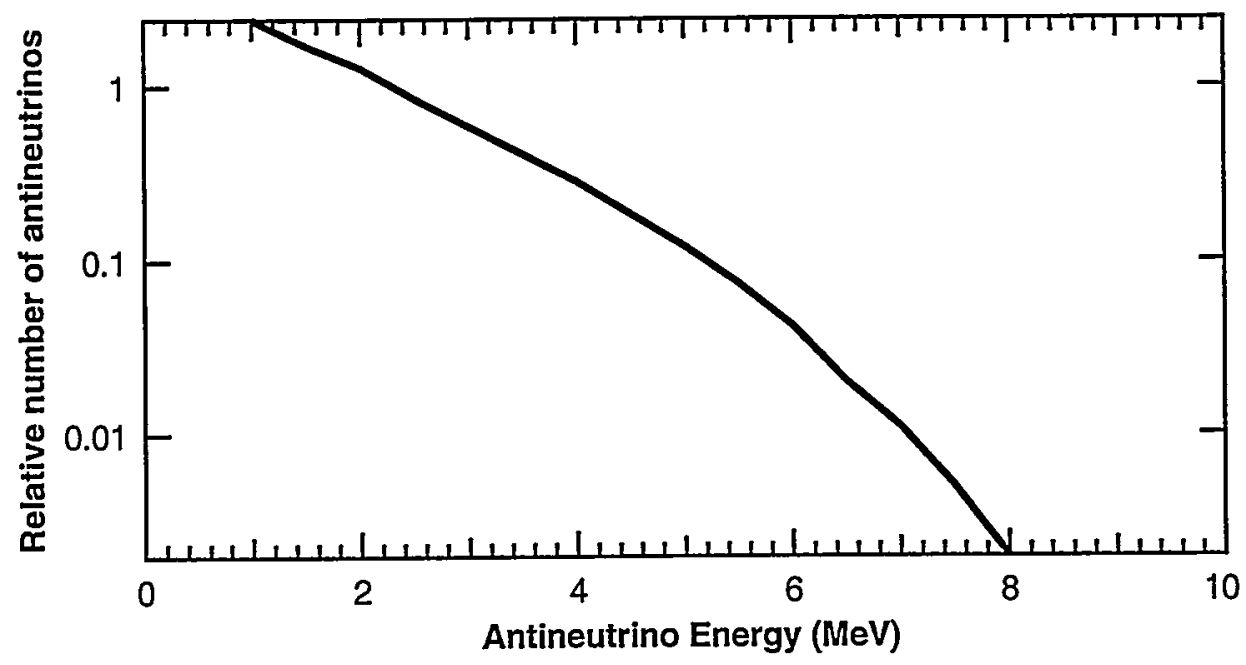

Figure 2: The approximate energy spectrum of antineutrinos produced in a U-235 fission explosion.

The time distribution of the antineutrino burst is set by the half-lives of the fission products. These peak at relatively short times, ranging from a few milliseconds to a few seconds, with a long tail extending out to very long half-lives. Figure 3 shows an approximate time distribution of the events. The mean lifetime is about 2.5 seconds.

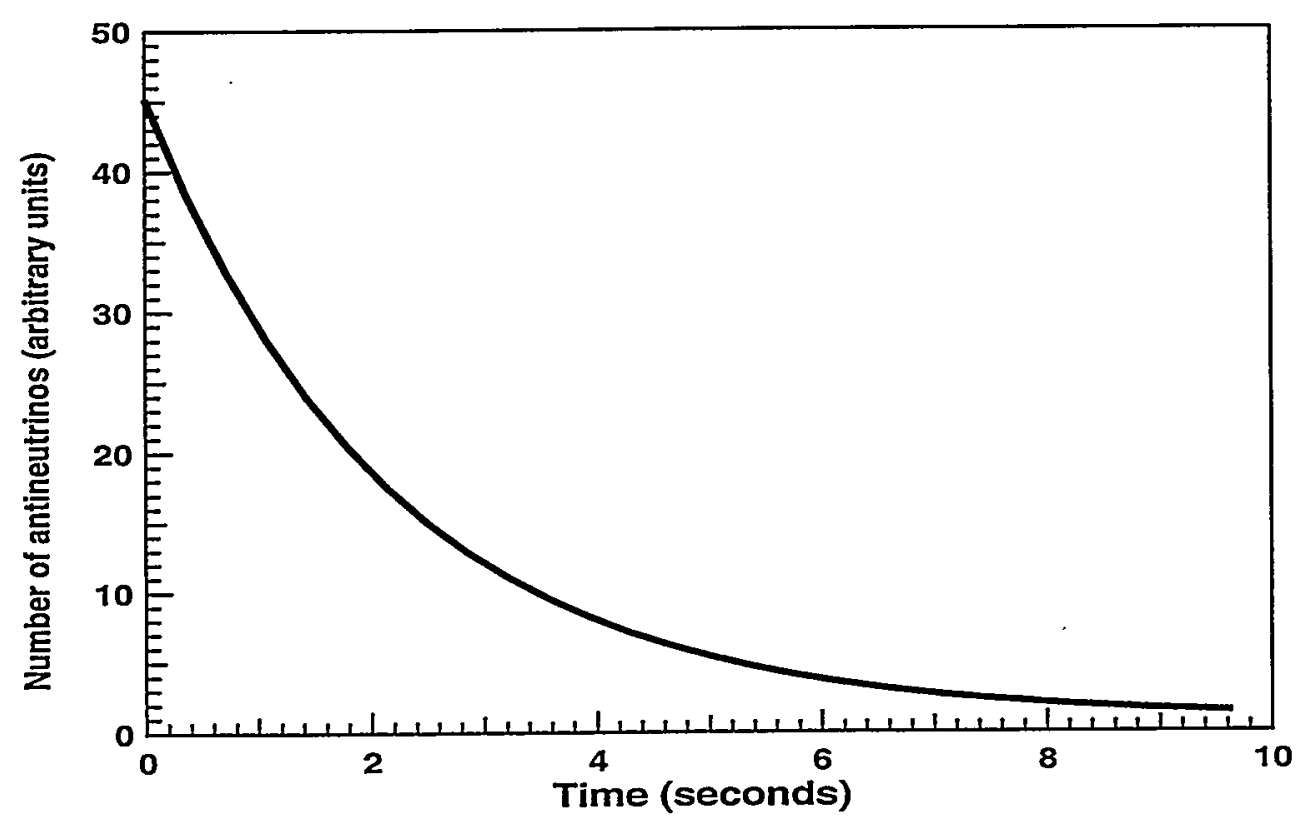

Figure 3: The approximate time distribution of the antineutrinos produced in a U-235 fission explosion.

In sum, a detector must be capable of registering antineutrino events with energies up to about $8 \mathrm{MeV}$ from a burst lasting a few seconds. 


\section{Antineutrino Interactions with Protons}

Antineutrinos are detected through interactions with quasi-free protons, with electrons, or with nuclei. Proton targets are well suited for detecting antineutrinos with energies in the $\mathrm{MeV}$ range because of the relatively high probability and low energy threshold of the interaction. In the proton-antineutrino interaction, referred to as inverse beta decay, the antineutrino converts the proton into a neutron and a positron, as shown in equation 2.

$$
\overline{\mathrm{v}}+\mathrm{p} \longrightarrow \mathrm{n}+\mathrm{e}^{+}
$$

The threshold $\bar{v}$ energy for this interaction is:

$$
1.8 \mathrm{MeV} \cong\left(\mathrm{m}_{\mathrm{n}}-\mathrm{m}_{\mathrm{p}}\right)+\mathrm{m}_{\mathrm{e}^{+}}
$$

where $m_{n}, m_{p}$, and $m_{e^{+}}$are the neutron, proton, and positron masses respectively.

Two target materials are most frequently used for detection of $\mathrm{MeV}$ scale antineutrinos in real time: $\mathrm{H}_{2} \mathrm{O}$ and liquid scintillator. The latter is preferable in terms of sensitivity and energy resolution, but too expensive and impractical for detectors containing more than a few thousand tons of material. ${ }^{11}$

The energy spectrum of antineutrinos produced by fission of ${ }^{239} \mathrm{Pu}$ and ${ }^{235} \mathrm{U}$, weighted by the inverse beta decay cross section is shown in Figure 4 . The rapidly decaying energy spectrum of the antineutrino is somewhat enhanced by an interaction probability that depends on the square of the antineutrino energy. As a result, the measured spectrum achieves a maximum at an antineutrino energy of about $4 \mathrm{MeV}$. The emitted positron has the same spectrum shifted to lower energies by $1.8 \mathrm{MeV}$.

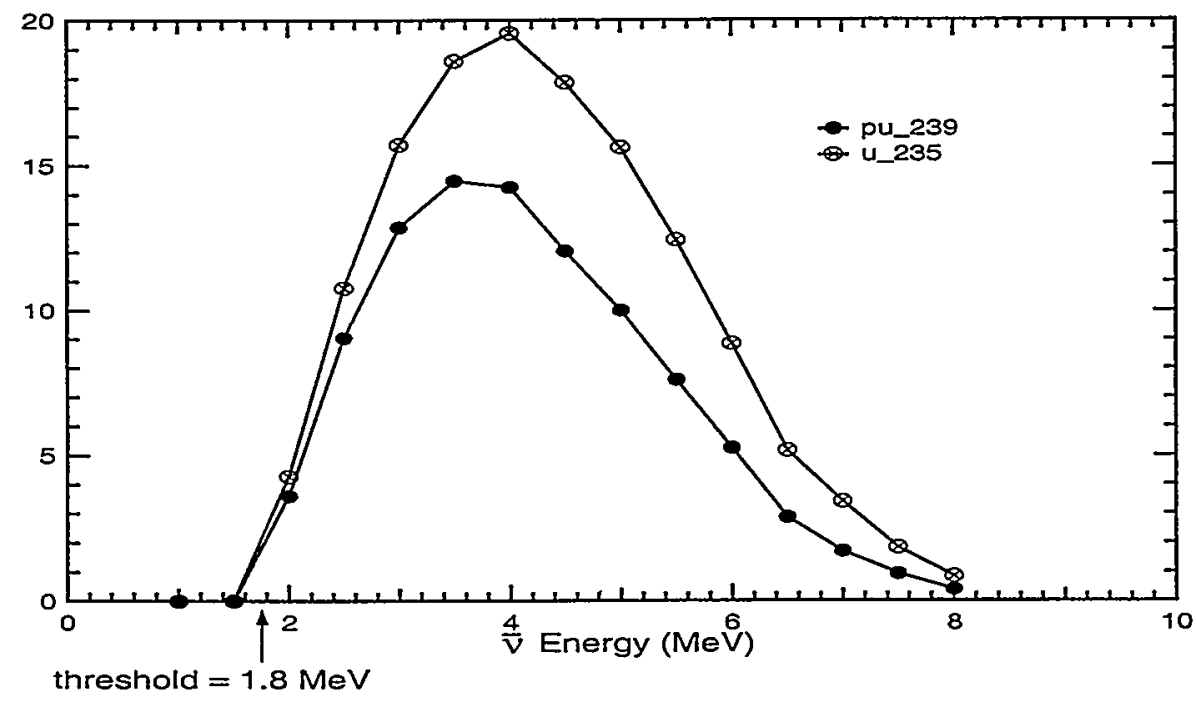

Figure 4: The energy spectrum of antineutrinos produced by the fission of $\mathrm{Pu}_{239}$ and $\mathrm{U}_{235}$ multiplied by the inverse beta decay cross section. The interaction threshold is $1.8 \mathrm{MeV}$. 


\section{The Number of Interactions in Fission Antineutrino Burst Detectors}

The size of the detector is fixed by requiring a minimum number of antineutrino interactions. As shown in equation 3 , the number of interactions is determined by the flux of incident antineutrinos, the proton-antineutrino interaction probability (the cross section), the density of free protons in the medium, and the detector volume.

3) $\quad \mathrm{N}_{\text {int }}=\frac{Y \cdot \mathrm{N}_{\text {fissions }}}{4 \pi \mathrm{r}^{2}} \cdot\left\{\int_{1.8 \mathrm{MeV}}^{\infty} \frac{\mathrm{dN}_{\overline{\mathrm{v}}}}{\mathrm{dE}_{\overline{\mathrm{v}}} \cdot \text { fission }} \cdot \sigma\left(\mathrm{E}_{\overline{\mathrm{v}}}\right) \cdot \rho \frac{\mathrm{N}_{\mathrm{Av}}}{\mathrm{A}} \mathrm{N}_{\text {free }} \cdot \mathrm{V}\right\}$

with

$\frac{d N_{\bar{v}}}{\mathrm{dE}_{\bar{v}} \cdot \text { fission }} \rightarrow$ the number of antineutrinos per fission emitted in an energy range $\mathrm{dE}_{\overline{\mathrm{v}}}$

$\mathrm{N}_{\mathrm{int}} \quad \rightarrow$ the number of $\overline{\mathrm{p}}$ interactions

$\mathrm{Y}(\mathrm{kt}) \quad \rightarrow$ the yield

$\mathrm{N}_{\text {fissions }} \rightarrow$ the number of fissions $/ \mathrm{kt}$

$\mathrm{r} \rightarrow$ the distance from the test to the detector

$\rho \quad \rightarrow$ the mass density

$\mathrm{N}_{\mathrm{Av}} \rightarrow$ Avogadro's constant

$\sigma\left(E_{\bar{v}}\right) \quad \rightarrow$ the $\bar{v} \mathrm{p}$ cross section at antineutrino energy $\mathrm{E}_{\bar{v}}$

A $\rightarrow$ the molecular weight

$N_{\text {free }} \rightarrow$ the number of free protons per molecule

$\mathrm{V} \rightarrow$ the detector volume

The lower limit of the integral is set by the interaction threshold of $1.8 \mathrm{MeV}$. The expression reduces to equation 4 , which relates the expected number of interactions to the yield of the fission explosion $Y$ in kilotons, the volume $V$ in cubic meters, and the distance from test to detector $r$ in meters. The coefficient $\alpha$, with units $(\mathrm{kt})^{-1} \mathrm{~m}^{-1}$, depends on the density of the detection medium as shown in Table 1.

$$
N_{\text {int }}=\frac{\alpha \cdot Y \cdot V}{r^{2}}
$$

Table 1: The constant $\alpha$ in equation 4.

\begin{tabular}{|l|l|l|l|}
\hline Medium & Water & Ice & Liquid Scintillator \\
\hline value of $\alpha\left(\times 10^{4}\right)$ & 5.4 & 5.0 & 4.24 \\
\hline
\end{tabular}

In conjunction with a minimum requirement for the total number of $\bar{v} p$ interactions, equation 4 can be used to generate approximate detector sizes suitable for use at various 
ranges. In the following sections, we describe the characteristics and estimate the size and sensitivity of liquid scintillator and $\mathrm{H}_{2} \mathrm{O}$ detectors. The similarity of the constant $\alpha$ between different media means that similar volumes of $\mathrm{H}_{2} \mathrm{O}$ and liquid scintillator are required to ensure a given number of interactions. However, liquid scintillator has far fewer backgrounds and higher energy resolution at $\mathrm{MeV}$ energies than $\mathrm{H}_{2} \mathrm{O}$.

Before proceeding to a discussion of particular antineutrino detectors, it is useful to provide a rough idea of the scales involved by calculating the volume needed to ensure at least two detected antineutrinos with $96 \%$ confidence from a $1 \mathrm{kt}$ nuclear explosion at a given distance. ${ }^{a}$

Table 2 shows this volume for distances ranging from $1 \mathrm{~km}$ to an Earth diameter (12800 $\mathrm{km}$ ). For comparison, the largest neutrino detector ever built, SuperKamiokande, contains 50,000 tons of purified water (equivalent to a cube $37 \mathrm{~m}$ on a side) and corresponding to a range of about $25 \mathrm{~km}$.

Table 2: The volume of water required to ensure at least one detected antineutrino with $95 \%$ confidence from a $1 \mathrm{kt}$ explosion at various distances from the explosion.

\begin{tabular}{|l|l|l|l|l|l|}
\hline $\begin{array}{l}\text { Distance from a 1 kt } \\
\text { test }(\mathbf{k m})\end{array}$ & 1 & 10 & 100 & 1000 & 12800 \\
\hline $\begin{array}{l}\text { Length (m) of the } \\
\text { edge of a cubic } \\
\text { volume required to } \\
\text { register a single } \\
\text { event with 95\% } \\
\text { confidence }\end{array}$ & 4 & 20 & 90 & 420 & 2300 \\
\hline
\end{tabular}

\section{Antineutrino Detection in Liquid Scintillator}

Antineutrinos interact with protons in liquid scintillator as described in equation 2 , producing a positron and a neutron. The positron deposits all of its energy within the scintillator volume, giving a prompt scintillation signal, including a $1.022 \mathrm{MeV}$ signal from its annihilation to two photons. The neutron will capture on proton targets after a mean time of about $150 \mu$ s (depending on the proton density of the scintillator) via the interaction:

5) $\mathrm{n}+\mathrm{p} \Rightarrow \mathrm{d}+\gamma(2.2 \mathrm{MeV})$

The photon produces visible ionization energy as it Compton scatters in the liquid scintillator.

${ }^{a}$ We take five interactions as the minimum number for detection of two events with $96 \%$ confidence. 
The neutron signal can be significantly enhanced and the capture time reduced by using a doping agent with a large thermal neutron capture cross-section, such as gadolinium, chlorine or lithium. ${ }^{12}$ Equation 6 shows the interaction in the case of gadolinium.

$$
\mathrm{n}+\mathrm{Gd} \Rightarrow \mathrm{Gd}^{*} \Rightarrow \mathrm{Gd}+\gamma^{\prime} \mathrm{s}(8 \mathrm{MeV})
$$

The neutron slows and is absorbed by the gadolinium about $30 \mu$ s after production, producing an excited nucleus that releases several $\mathrm{MeV}$ of energy in a photon shower. The exact time delay and energy release depends on the element and concentration, with concentrations in existing experiments ranging from 0.1 percent for gadolinium to 2.5 percent for sodium chloride, another effective neutron absorber.

Photomultiplier tubes are used to detect and amplify the visible light emitted as the positron and the neutron-capture gamma rays ionize in the liquid scintillator. The light output is high: a few thousand photons per $\mathrm{MeV}$ of deposited energy. The high light yield of the scintillator and the sharp time correlation of the positron and neutron signals allows for effective rejection of backgrounds, even for the relatively low energy (MeV scale) antineutrinos produced by fission.

\section{Design and Sensitivity of Liquid Scintillator Detectors}

Here we use design parameters and background estimates for the 1000 ton KamLAND liquid scintillator detector, currently being built in the Kamioka mine site in Japan. ${ }^{13}$ This type of detector is well suited to our purpose, because it is designed for the detection of fission antineutrinos (from reactors) with energies in the same range as those produced by a nuclear explosion. The largest liquid scintillator detector now in operation is the 1600 ton Large Volume Detector (LVD) at the Gran Sasso Laboratory in Italy. ${ }^{14}$

The KamLAND detector, shown in Figure 5, consists of a sphere of liquid scintillator, surrounded by neutron and gamma ray absorbing buffers made of liquid paraffin and water. The liquid scintillator is enclosed in a transparent nylon composite balloon that has low permeability to radon, a background-generating contaminant. The total mass of liquid scintillator is 1000 tons, filling a sphere with a radius of about $6.3 \mathrm{~m}$. A 500 ton fiducial spherical volume is defined within the main volume to help reduce backgrounds from photomultiplier tubes and from external radioactive decay sources. The liquid paraffin buffer is enclosed in a spherical stainless steel vessel, which is in turn surrounded by a water Cerenkov veto counter. The entire detector is shielded by a rock overburden of 2700 meters of water equivalent (m.w.e.). 


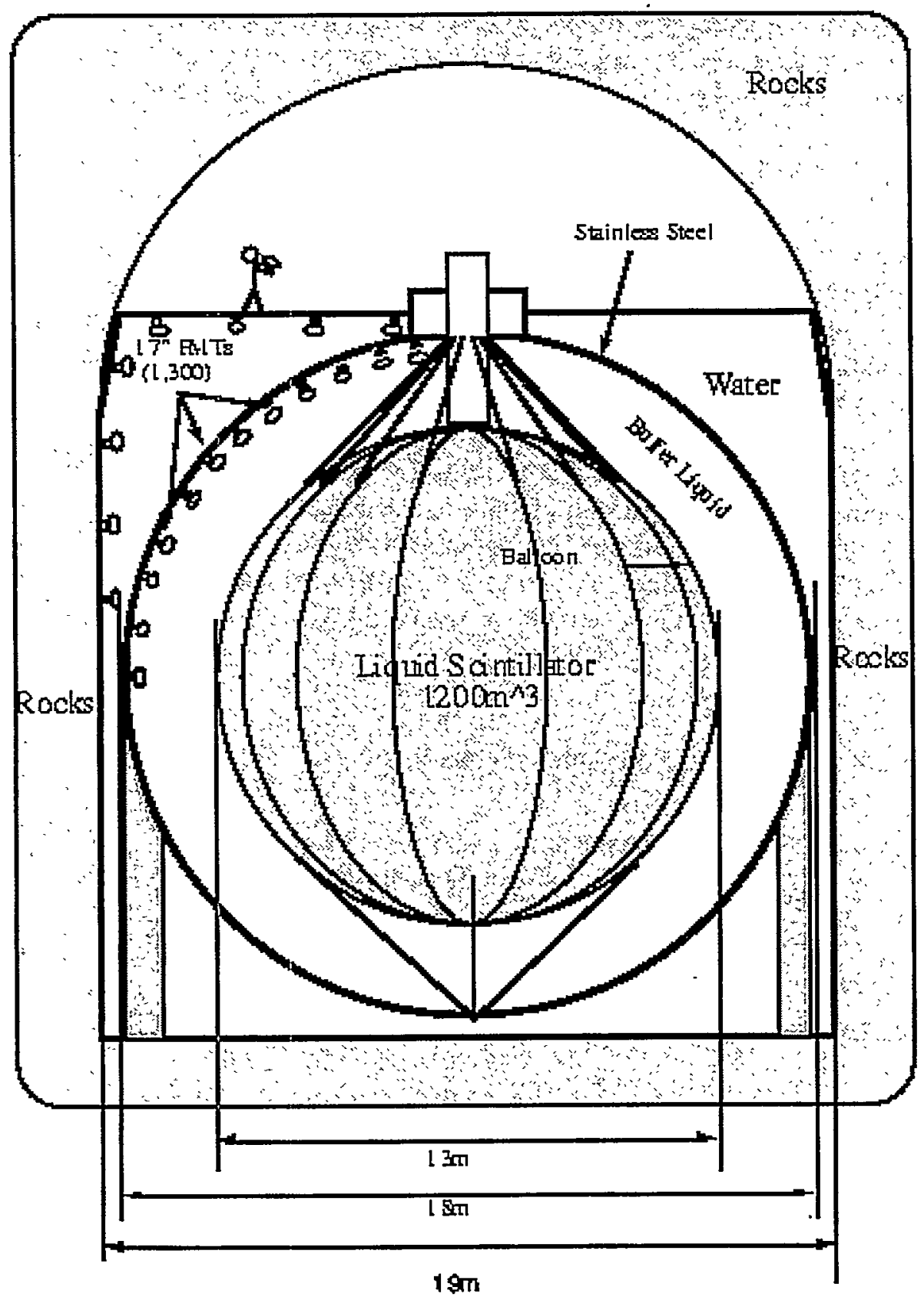

Figure 5: The KamLAND liquid scintillator detector. (Picture from reference 13.)

The visible light produced by positron decay and neutron capture is recorded using 1300 photomultiplier tubes. Events are selected according to the magnitude of the energy depositions of the positron and neutron, and the spatial and time coincidence between the positron and neutron. The spatial reconstruction resolution is an estimated $10 \mathrm{~cm}$ for a 1 $\mathrm{MeV}$ event, while the time resolution of the phototubes is less than $10 \mathrm{~ns}$. 
To estimate background rates, we must choose specific selection criteria. The cuts listed below are taken from reference 13 .

7)

$$
\begin{aligned}
& \mathrm{E}_{\text {positron }}>3 \mathrm{MeV} \\
& 1.8 \mathrm{MeV}<\mathrm{E}_{\text {neutron }}<2.6 \mathrm{MeV} \\
& 10 \mu \mathrm{s}<\Delta \mathrm{t}<500 \mu \mathrm{s} \\
& |\Delta \overrightarrow{\mathrm{x}}|<1 \mathrm{~m}
\end{aligned}
$$

with the variables

$$
\begin{aligned}
& \mathrm{E}_{\text {positron }} \rightarrow \text { the reconstructed positron energy } \\
& \mathrm{E}_{\text {neutron }} \rightarrow \text { the reconstructed neutron energy } \\
& \begin{array}{ll}
\Delta \mathrm{t} & \rightarrow \text { the time delay between the positron and neutron } \\
\Delta \overrightarrow{\mathrm{x}} & \rightarrow \text { the vector difference between the positron and neutron positions }
\end{array}
\end{aligned}
$$

\section{Detection Efficiency}

The detection efficiency is affected by two main factors. First, the 500 ton fiducial volume is only 50 percent of the total liquid scintillator volume, in order to attenuate radioactivity produced by photomultiplier tubes and by external rock. Second, the three $\mathrm{MeV}$ energy threshold for the positron removes about 65 percent of the signal, for an approximate overall detection efficiency of 20 percent.

\section{Non-Antineutrino Backgrounds}

There are two types of non-antineutrino backgrounds to the events just defined:

1. Uncorrelated events, in which the signal is mimicked by accidental coincidences between independent positron and neutron-like signals, or "singles", produced by ambient radioactivity and cosmic rays.

2. Correlated events, in which the signal is mimicked by a single interaction that produces both a positron and neutron-like signal within the appropriate time window. Correlated events arise primarily from muon capture or muon spallation on nuclei in or near the detector. These processes produce high energy neutrons which first strike target protons to give a signal similar to that of the positron, and then produce the coincident neutron signal upon absorption. In addition, some correlated events arise from the cosmogenic production of nuclei such as ${ }^{11} \mathrm{Li}$ and ${ }^{9} \mathrm{Li}$, which can also emit correlated positron and neutron-like signals in the appropriate energy range. 


\section{Uncorrelated Backgrounds}

The most important radionuclide backgrounds are beta and gamma decays from ${ }^{238} \mathrm{U}$, ${ }^{232} \mathrm{Th}$ decay products (including radon). Decays emanating from the recessed phototubes and from external rock are largely shielded by the buffer. Backgrounds produced within the buffer and within the liquid scintillator itself must be reduced by purification.

Assuming radiopurity levels of uranium and thorium at the level of $10^{-14}$ grams per gram of liquid scintillator $(\mathrm{g} / \mathrm{g})$ and a radon concentration of $0.5 \mathrm{mBq} / \mathrm{m}^{3}$, the accidental coincidence rate $\mathrm{r}_{\mathrm{acc}}$ is given by:

8)

$$
r_{\text {acc }}=r_{1} r_{2} \tau \frac{V_{\text {event }}}{V_{\text {fiducial }}}
$$

with

$$
\begin{array}{ll}
\mathrm{r}_{1}=0.04 \mathrm{~Hz} & \rightarrow \text { the positron-like singles rate } \\
\mathrm{r}_{2}=1.5 \mathrm{~Hz} & \rightarrow \text { the neutron-like singles rate } \\
\tau=500 \mu \mathrm{sec} & \rightarrow \text { the PMT time gate } \\
\mathrm{V}_{\text {event }} \cong 4 \mathrm{~m}^{3} & \rightarrow \text { the containment volume for the event } \\
\mathrm{V}_{\text {fiducial }}=700 \mathrm{~m}^{3} & \rightarrow \text { the fiducial volume of the detector }
\end{array}
$$

The coincidence rate of the two signals within a $500 \mu$ s time gate is no more than 0.015 events per day. At the KamLAND depth of 2700 m.w.e., $1.4 \times 10^{-4}$ events per day, or less than one percent of the uncorrelated background, is due to cosmic rays.

\section{Correlated Backgrounds}

Correlated backgrounds are produced primarily by cosmic muon interactions. The rate of muons is only about $0.3 \mathrm{~Hz}$ in the KamLAND detector. An estimated upper bound on the rate of cosmogenic correlated events is about 0.013 per day in the fiducial volume, with the estimate applicable for positron energies in the $1-2.7 \mathrm{MeV}$ range. The actual background will be significantly lower than this number, because of the $3 \mathrm{MeV}$ positron energy threshold in the selection criteria. ( See Equation 7.)

\section{Total Correlated and Uncorrelated Non-Antineutrino Backgrounds}

In all, the total background in the KamLAND detector is estimated to be below 0.028 events per day, or about 10 per year. We note that the accidental coincidence rate estimates given here do not include the use of gadolinium. If gadolinium were added at

\footnotetext{
${ }^{\mathrm{a}} 1 \mathrm{~Bq}=1$ disintegration/second.
} 
the 0.1 percent level, the neutron capture time would decrease to under $30 \mu \mathrm{s}$, and the uncorrelated background rate would decrease by a factor of about five.

\section{Antineutrino Backgrounds}

The three largest sources of real antineutrino background are supernovae, geological antineutrinos and reactor antineutrinos. All are small (or can be made small) in the energy range of interest compared to the non-antineutrino backgrounds just described.

A supernova near the center of the Milky Way, about 30,000 light years from the Earth, with a total energy of $10^{53}$ ergs is expected to produce several hundred $20-30 \mathrm{MeV}$ antineutrino events over a 10 second period in a 1000 ton liquid scintillator detector. ${ }^{15}$ Frequency estimates are in the neighborhood of a few per century. ${ }^{16}$ Even if the supernova rate is higher than anticipated, the event could be distinguished from fission burst by its significantly higher energy spectrum and by associated bursts in other antineutrino detectors and possibly telescopes. So far, one supernova (SN1987A) has been detected via an antineutrino burst in two detectors, accompanied by a visible light signature.

Antineutrinos emanate from the Earth, primarily from beta decays in the uranium and thorium decay chains. The total signal is estimated to be 50 to $150 /$ year for a 1000 ton detector. ${ }^{17}$ The maximum geologic antineutrino energy is $3.27 \mathrm{MeV}$, corresponding to a $2.5 \mathrm{MeV}$ energy deposition ${ }^{\mathrm{a}}$ in the detector. Virtually all of these low energy events will be removed by the $3 \mathrm{MeV}$ cut on the deposited positron energy.

Reactor antineutrinos have nearly the same energy spectrum as fission explosion antineutrinos. The KamLAND detector, at a distance of about 100-300 km from all Japanese reactors, anticipates a total signal of $\sim 750$ antineutrino events per year from reactors with a total of $130 \mathrm{GW}$ of thermal power. This background can be made small by building the detector far from reactor sites. Relative reactor antineutrino fluxes at various locations in the world, calculated by Rothschild et al., ${ }^{18}$ shows the KamLAND site as having a reactor antineutrino rate 30 to 100 times higher than more remote areas, such as Hawaii, Tibet, and central Australia. In the next section we scale the non-antineutrino backgrounds to shallower depths, in which case a reactor background of 100 events per year is small relative to muon-induced backgrounds.

\section{Scaling Backgrounds to Shallower Depths}

It is useful to extrapolate the cosmic ray components of the background in the deep KamLAND detector to shallower depths at which detector construction is more feasible. Most of the cosmic ray induced background comes from neutrons with energies up to several hundred $\mathrm{MeV}$ produced by muon capture and spallation in materials surrounding the detector. Few experiments have been done that give detailed information on the

\footnotetext{
${ }^{a} \mathrm{E}-1.8 \mathrm{MeV}+2 \mathrm{~m}_{e}$, with $\mathrm{E}$ the antineutrino energy and $\mathrm{m}_{\mathrm{e}}$ the electron mass.
} 
energy spectrum of such neutrons. Roughly, however, the scaling will depend on the product of the total muon generated neutron production rate and the cosmic muon intensity at each depth.

Figure 6 shows the variation in high energy neutron production with depth. The curve is derived from an approximate table of neutron production rates per cosmic muon versus depth as measured by Aglietta et al. ${ }^{19}$ multiplied by the relative number of cosmic muons at the same depth.

Relative number of neutrons

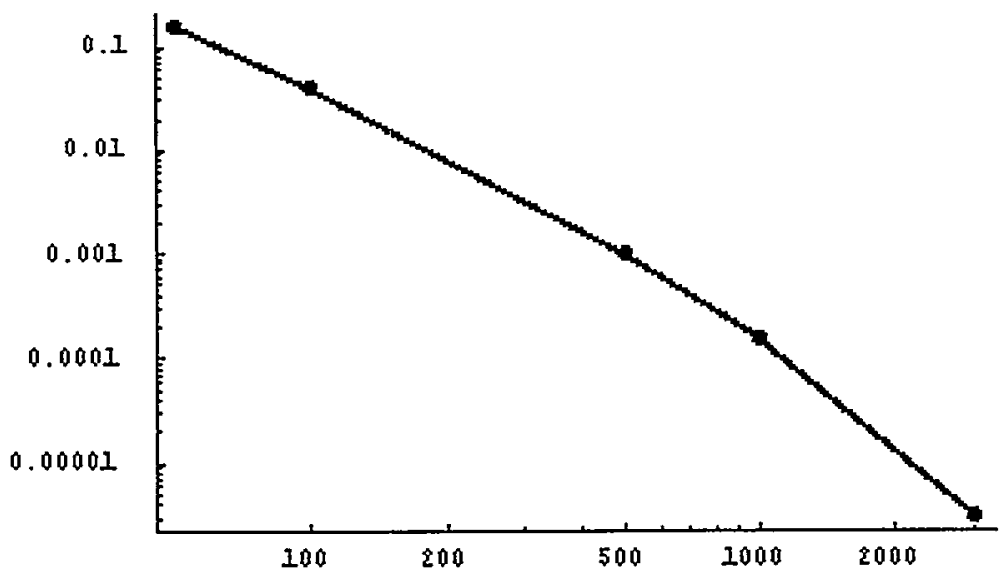

Depth (m.w.e.)

Figure 6: The relative number of neutrons produced by cosmic muons versus depth in m.w.e.

Using this curve as a guide, the correlated background scaled to a depth of $50 \mathrm{~m}$.w.e. is about 79 events per day in a 1000 ton detector. This is an upper bound on the correlated background because of the $3 \mathrm{MeV}$ energy threshold for the positron signal. The cosmic ray associated uncorrelated background rate, scaled to a depth of 50 m.w.e., is only about 1 event/day. The total background at $50 \mathrm{~m}$.w.e. is therefore less than 80 events per day, while at $100 \mathrm{~m}$.w.e., the total background is less than 10 events per day. Both of these rates are large compared to the reactor antineutrino background of less than 1 event per day.

\section{Fixing the Number of Events, Detector Size, and Test-Detector Distance}

The volume of the detector is determined directly from equation 4 once the desired number of antineutrino events is fixed. Since the volume is directly proportional to the number of events, it is important to minimize the number of events required for definitive evidence of an explosion. The minimum signal strength can be set by assuming a Poisson distributed background and demanding that it produce no more than one false positive event per century. (Below we will see that relaxing this strict criterion to once per year 
has only a modest effect on the minimum signal level.) The calculations here use the total background estimate for a detector at a depth of 50 m.w.e. in a 10 second interval. For a detector at this depth, the number of background events is:

$\lambda=0.01$ events (per kt of liquid scintillator in a 10 second interval)

The background restriction is expressed as:

9) $\quad 1-\sum_{\mathrm{p}=0}^{\mathrm{p}=(\mathrm{m}-1)} \frac{\mathrm{e}^{-\lambda} \lambda^{\mathrm{p}}}{\mathrm{p} !} \leq \frac{10 \mathrm{sec}}{100 \mathrm{yr} \cdot 3.15 \times 10^{7} \mathrm{sec} / \mathrm{yr}}$

with

$\mathrm{m} \quad \rightarrow$ the number of signal events

Using this criterion, Table 3 shows the number of events required in a KamLAND-sized (1000 ton) detector, and in a set of five such detectors (total mass 5000 tons), as well as the distance at which detectors of the given size would be sensitive to this number of events. The number of events has been scaled upward to reflect the estimated 20 percent detection efficiency. The range for the large 5000 ton detector array could be extended to about $6.5 \mathrm{~km}$ with 100 percent signal detection efficiency. Relaxing the criterion to allow one false positive per year reduces the number of required events for a $1000(5000)$ ton detector to $15(20)$, and gives only a modest increase in the maximum distance for sensitivity to a $1 \mathrm{kt}$ explosion.

Table 3: The number of raw antineutrino interactions required to guarantee no more than one background-generated false positive $1 \mathrm{kt}$ event per century. Also shown is the distance from a $1 \mathrm{kt}$ nuclear test at which this mass of scintillator is sensitive to the indicated number of events, as derived from equation 4 . The background estimate has been scaled to a depth of 50 m.w.e.

\begin{tabular}{|l|l|l|}
\hline $\begin{array}{l}\text { Detector mass } \\
\text { (tons) }\end{array}$ & $\begin{array}{l}\text { Number of raw events } \\
\text { required to guarantee less } \\
\text { than one false positive per } \\
\text { century }\end{array}$ & $\begin{array}{l}\text { Maximum distance } \\
\text { from a 1 kt explosion } \\
\text { for sensitivity to this } \\
\text { number of events }\end{array}$ \\
\hline 1000 & 20 & $1.5 \mathrm{~km}$ \\
\hline 5000 & 25 & $3 \mathrm{~km}$ \\
\hline
\end{tabular}

In summary, liquid scintillator detectors operating a few tens of meters below the Earth, with sizes and characteristics similar to existing detectors, could detect $1 \mathrm{kt}$ explosions at a range of a few kilometers. 


\section{Antineutrino Detection in $\mathrm{H}_{2} \mathrm{O}$}

The cut-off range for the use of scintillator is a few kilometers, due to the prohibitive cost and environmental impact associated with the huge amounts of scintillator required at greater distances. Beyond this range, water is probably the only suitable medium, despite the inferior sensitivity of water based detectors to low energy antineutrinos.

Water is less sensitive than liquid scintillator for two reasons. First, there is far less light output per unit of deposited energy, since detection depends on measuring relatively weak Cerenkov light rather than the much stronger ionization signal seen in liquid scintillator. Second, the neutron signal is normally not measured in $\mathrm{H}_{2} \mathrm{O}$, so the spatial and time correlation between the positron and neutron cannot be used for noise rejection. As a result, the background rate is much higher than in liquid scintillator, consisting of any cosmic rays and ambient radioactivity that produce positron-like signals in the few $\mathrm{MeV}$ range. Because of these drawbacks, water Cerenkov detectors have so far been limited to detection of neutrinos with energies above an approximate five $\mathrm{MeV}$ threshold. The five $\mathrm{MeV}$ threshold is difficult to lower, as fission burst detection would require.

Backgrounds might be reduced dramatically if the neutron could be detected in coincidence with the positron. This could be done by using a dopant with a high neutron absorption probability such as $\mathrm{NaCl}, \mathrm{MgCl}$, or $\mathrm{Gd}$. This is an option being considered for the SNO heavy water detector, ${ }^{20}$ in which 2.5 tons of $\mathrm{NaCl}$ is to be dissolved in 1000 tons of $\mathrm{D}_{2} \mathrm{O}$. It has never been done on the scales contemplated here. The advantage of neutron recovery derives from the fact that only the relatively rare time-coincident backgrounds would survive event selection.

Below we develop approximate limits on sensitivity and range for water Cerenkov detectors with and without dopants. We begin by estimating the size and phototube requirements for long range fission burst detectors, followed by a discussion of backgrounds. Next, we show that background restrictions severely limit the prospects for fission burst detection at ranges much beyond $10 \mathrm{~km}$ without the addition of a dopant. We then demonstrate that the sensitive range for a $1 \mathrm{kt}$ test might be extended to about $1000 \mathrm{~km}$ through the use of a dopant, taking gadolinium as an example.

\section{Detecting a Fission Antineutrino Burst in $\mathrm{H}_{2} \mathrm{O}$}

Water detectors measure the Cerenkov light produced as the positron from the inverse beta decay process (equation 2) slows in the medium. The most probable positron energy is about two $\mathrm{MeV}$, corresponding to the peak antineutrino energy of about $3.8 \mathrm{MeV}^{\mathrm{a}} \mathrm{A}$ two $\mathrm{MeV}$ positron travels about $1 \mathrm{~cm}$ through $\mathrm{H}_{2} \mathrm{O}$, producing about 270 Cerenkov photons in the 350 to $550 \mathrm{~nm}$ range which may be detected by phototubes. ${ }^{21}$ For

\footnotetext{
${ }^{a}$ We neglect the small amount of Cerenkov light arising from Compton scattering of the positron annihilation photons.
} 
comparison, several thousand visible photons per $\mathrm{MeV}$ are produced by ionization in liquid scintillator.

The Cerenkov photons are emitted in a cone with an opening angle of about 40 degrees. This angle is increased by a comparable amount due to multiple scattering of the low energy positrons. As long as event shape algorithms are not relied upon to select events, the spherical detector geometry we will adopt below is insensitive to the details of the scattering process.

As previously mentioned, the detector size is set by specifying a target number of events per unit of yield at a specified range. Ideally, the number of events should be optimized as a function of the background. We set the number of events to be 10 , large enough so that the burst is relatively difficult to reproduce by background fluctuations, but as small as possible to minimize the required number of modules. A 5 event pulse is an absolute lower limit on the acceptable signal size, since below this number downward Poisson fluctuations in the signal could cause real events to be missed more than 5 percent of the time.

\section{Design Considerations}

Because of the small number of photons produced per event, a large number of phototubes and as hermetic a detector as possible are required for efficient recovery of the signal. For reasons of simplicity and efficiency, we consider a spherical array of phototubes surrounding a central volume of water.

The radius of the sphere is constrained by the need to reduce the spread in photon arrival times at phototubes and the effect of light attenuation on the signal. A large spread in arrival times increases the length of time over which phototube pulses must be summed, and hence the gated noise. Above a $200 \mathrm{~ns}$ time difference, the phototube noise becomes intolerable. In the following treatment we use modules with total diameters of $50 \mathrm{~m}$, with events accepted in a $45 \mathrm{~m}$ diameter inner sphere, corresponding to a $200 \mathrm{~ns}$ time window for collection of the signal by phototubes. ${ }^{\text {a }}$ This diameter allows the use of phototube with noise rates ("dark current") of a few $\mathrm{kHz}$, a level compatible with that of existing tubes. (See Appendix I.) In purified water, the attenuation length for blue Cerenkov light is about $60 \mathrm{~m}$. Significantly larger radius detectors would therefore result in an unacceptable loss of signal. The total amount of water in a $50 \mathrm{~m}$ diameter spherical module is 65,550 tons, while the $45 \mathrm{~m}$ diameter fiducial volume of the detector contains 47,712 tons of water. This is comparable to the total mass of the SuperKamiokande detector $(50,000$ tons), the largest purified water Cerenkov detector ever built.

The range of a $50 \mathrm{~m}$ radius module for a $1 \mathrm{kt}$ explosion is only a few tens of $\mathrm{km}$ depending on the number of events required in the signal. To reach greater distances, we must use arrays of modules. The signal at long distances then consists of a single antineutrino event in each of a subset of modules in the array, all occurring within a 10 second time gate.

\footnotetext{
${ }^{a}$ At the ten kilometer range, a slightly smaller 40 meter diameter module is adequate.
} 


\section{Light Yield for an Antineutrino Event}

The positron from the antineutrino interaction is produced anywhere in the detector volume with equal probability. Since the positron travels only a few $\mathrm{cm}$ before decay, the photon source is approximately point-like on the scale of the detector. With this assumption, the probability that an interaction occurs at a radial distance $\mathrm{x}$ from the surface of a sphere of radius $r$ is given by:

$\mathrm{P}(\mathrm{x})=\frac{3(\mathrm{r}-\mathrm{x})^{2}}{\mathrm{r}^{3}}$

Accounting for attenuation, the mean number of photons per interaction per $\mathrm{MeV}$ is:

10) $\mathrm{N}_{\mathrm{pe}}=\mathrm{N}_{\gamma} \eta \frac{\mathrm{N}_{\mathrm{PMT}} \mathrm{A}_{\mathrm{PMT}}}{4 \pi \mathrm{r}^{2}} \int_{0}^{\mathrm{r}} \mathrm{P}(\mathrm{x}) \mathrm{e}^{\frac{-\mathrm{x}}{\lambda}} \mathrm{dx}=\frac{12}{\mathrm{MeV}}$

with

$\mathrm{N}_{\gamma}=135 \rightarrow$ the number of Cerenkov photons per $\mathrm{MeV}$

$\eta=0.2 \quad \rightarrow$ the photocathode quantum efficiency

$\mathrm{N}_{\mathrm{PMT}} \rightarrow$ the number of phototubes

A PMT $\rightarrow$ the photocathode area

$\mathrm{r}=25 \mathrm{~m} . \quad \rightarrow$ the detector radius

$\lambda=60 \mathrm{~m}$. $\rightarrow$ the attenuation length in purified water

With this configuration, a typical $\sim 2.0 \mathrm{MeV}$ positron produced by the fission burst will therefore trigger about 25 phototubes on average. A single phototube will rarely see more than 1 photoelectron. The ratio $\left(\mathrm{N}_{\mathrm{PMT}} \mathrm{A}_{\mathrm{PMT}}\right) /\left(4 \pi \mathrm{r}_{\mathrm{det}}{ }^{2}\right)$ is taken to be 0.5 , a little larger than the ratio in existing experiments of about 0.4 .

\section{Array Size and Phototube Requirements}

Using the geometry and photostatistics just defined, we can estimate the number of modules and phototubes required for sensitivity to a $1 \mathrm{kt}$ test at a given distance. Once these parameters are determined, the tolerable noise level can be set by demanding that a false positive occur no more than once per century.

The size of the detector is fixed by the number of desired events, assumed to be 10 from a $1 \mathrm{kt}$ explosion. To ensure a reasonable number of photoelectrons, we demand a minimum positron energy of $2.0 \mathrm{MeV}$, resulting in a signal loss of about 38 percent. The detector volume is scaled upward to compensate for the efficiency loss.

At a range of $10 \mathrm{~km}$, a sphere 40 meters in diameter is large enough to register 10 events (above a $2 \mathrm{MeV}$ positron energy threshold) from a $1 \mathrm{kt}$ explosion. Assuming a photocathode area $A_{\mathrm{PMT}}$ of about $0.2 \mathrm{~m}^{2}$, about 12,800 phototubes are required for 50 percent coverage of the sphere's surface. 
At distances of $100 \mathrm{~km}$ and above, an array of larger $45 \mathrm{~m}$ diameter modules would be required to register 10 events from a $1 \mathrm{kt}$ explosion. Table 4 shows the number of modules, number of phototubes per module, and total number of phototubes required as a function of detector range. For comparison, the number of phototubes in a modern large scale physics experiment is around 10,000, and the number of independent electronics channels around 100,000. Reductions in the numbers shown in Table 4 by a factor of two or three might be possible with increases in photocathode efficiency, or with improved light collection methods such as focusing lenses or mirrors. Absent such improvements and barring major price reductions, the required number of photodetectors is unattainable beyond about the $100 \mathrm{~km}$ range.

Table 4: The number of modules of the indicated size required to detect a 10 event burst from a $1 \mathrm{kt}$ explosion at the indicated distance. Also shown are the number of phototubes per module and total number of phototubes at each range.

\begin{tabular}{|c|c|c|c|c|}
\hline $\begin{array}{c}\text { Range } \\
\text { (distance from } \\
\text { test to detector, } \\
\mathrm{km} \text { ) }\end{array}$ & $\begin{array}{c}\text { Module radius } \\
(\mathbf{m})\end{array}$ & $\begin{array}{c}\text { Number of } \\
\text { modules per } \\
\text { detector }\end{array}$ & $\begin{array}{c}\text { Number of } \\
\text { PMTs per } \\
\text { module }\end{array}$ & $\begin{array}{c}\text { Total } \\
\text { number of } \\
\text { PMTs }\end{array}$ \\
\hline $\mathbf{1 0}$ & 20 & 1 & 12800 & 12800 \\
\hline $\mathbf{1 0 0}$ & 22.5 & 63 & 16200 & $\sim 10^{6}$ \\
\hline $\mathbf{1 0 0 0}$ & 22.5 & $\sim 6300$ & 16200 & $\sim 10^{8}$ \\
\hline $\begin{array}{c}\sim \mathbf{1 2 , 8 0 0} \text { (Earth } \\
\text { Diameter) }\end{array}$ & 22.5 & $\sim 10^{6}$ & 16200 & $\sim 10^{10}$ \\
\hline
\end{tabular}

\section{Module Noise Restrictions}

At distances of $100 \mathrm{~km}$ and above, the signal consists of a single antineutrino event in each of 10 detectors in a 10 second time gate. Actual burst-like backgrounds such as supernovae are low, while multiple events produced in a single module from muon showering would not satisfy the multiple module requirement. Therefore the only events of concern are accidental fluctuations to the event level in multiple modules within the 10 second time window. To derive an upper limit on acceptable noise levels per module, we calculate the probability that false positives occur in any $\mathrm{m}(=10)$ out of $\mathrm{n}$ detectors in a 10 second period, and demand that this probability be small. $\mathrm{m}$ and $\mathrm{n}$ can be related by modifying equation 4 : 
11) $\mathrm{n}=\frac{\mathrm{V}_{\text {array }}}{\mathrm{V}_{\text {mod }}}=\frac{\mathrm{m} \cdot \mathrm{r}^{2}}{\mathrm{Y} \alpha \varepsilon \mathrm{V}_{\mathrm{mod}}}$;

with

$\mathrm{n}$

$\mathrm{m}(=10)$

r

$\alpha=54000 \mathrm{kt}^{-1} \mathrm{~m}^{-1}$

$\varepsilon=0.62$

$\mathrm{Y}(\mathrm{kt})$

$\mathrm{V}_{\text {mod }}$

$\mathrm{V}_{\text {array }}$ $\rightarrow$ the number of modules required to ensure $m$ interactions

$\rightarrow$ the number of modules that trigger over a 10 second time period due to the fission burst

$\rightarrow$ the test to detector distance

$\rightarrow$ the strength constant for water

$\rightarrow$ the detection efficiency

$\rightarrow$ the yield

$\rightarrow$ the volume of a single module

$\rightarrow$ the total detector volume

The number of accidental coincidences $\lambda$ of any set $m$ of the $n$ modules in time $\tau$ is:

$$
\lambda=\mathrm{R}_{\mathrm{acc}}^{\mathrm{m}} \tau=\mathrm{C}(\mathrm{n}, \mathrm{m}) \times \mathrm{m} \times\left(\mathrm{R}_{\bmod } \tau\right)^{\mathrm{m}} ; \mathrm{R}_{\bmod } \tau<<1
$$

where

$$
\begin{array}{ll}
\mathrm{C}(\mathrm{n}, \mathrm{m})=\mathrm{n} ! /(\mathrm{m} !(\mathrm{n}-\mathrm{m} !)) & \rightarrow \text { the binomial coefficient } \\
\tau=10 \mathrm{~s} & \rightarrow \text { the duration of the burst } \\
R_{\mathrm{mod}} & \rightarrow \text { the background rate per module } \\
\mathrm{R}_{\mathrm{acc}}^{\mathrm{m}} & \rightarrow \text { the rate of accidental coincidences in } \mathrm{m} \text { out of } \mathrm{n} \\
& \text { modules }
\end{array}
$$

The overall coincidence rate described by equation 12 places an upper limit on $R_{\text {mod }}$, the tolerable noise rate per module. This limit obtains whether the signal is assumed to be the positron only, or a correlated positron-neutron pair. Thus the upper limit can be applied to detectors with and without a dopant.

Table 5 shows the noise rates per module which ensure that a fluctuation to the 10 event level in any 10 second period in the entire array occurs less than once per century. Comparison of these target event rates with various background estimates allows restrictions to be placed on the range, contamination levels and construction depth of fission burst detectors. As before, relaxing the background restriction to allow one false positive per year rather than per century has only a modest effect on the tolerable background noise level. For example, the allowable noise at $1000 \mathrm{~km}$ becomes 1.1 events per day instead of 0.7 . 
Table 5: The background rate per module required for sensitivity to 10 events from a $1 \mathrm{kt}$ test in a water-based detector at the indicated distance, with the constraint of no more than one false positive per century.

\begin{tabular}{|c|c|}
\hline $\begin{array}{l}\text { Range } \\
\text { (distance from test to detector, } \mathrm{km} \text { ) }\end{array}$ & $\begin{array}{l}\text { Noise rate per module that ensures } \\
\text { no more than one false positive per } \\
\text { century (events/day) }\end{array}$ \\
\hline 10 & 5875 \\
\hline 100 & 75 \\
\hline 1000 & 0.7 \\
\hline$\sim 12,800$ (Earth Diameter) & 0.004 \\
\hline
\end{tabular}

\section{Backgrounds}

In the previous section we estimated the upper limit on noise from all sources for a single module, based on a 10 event signal. The main backgrounds arise from uranium and thorium decays in the active medium, detector walls, photomultiplier tubes, and surrounding rock, from proton recoil due to muon generated fast neutrons, and from activation of nuclei by fast neutrons and muons.

Without a dopant, these backgrounds are much more severe in water because of the absence of a coincident neutron signal. Additionally, phototube dark current fluctuations and solar neutrinos are indistinguishable on an event by event basis from the signal, although the muogenic and ambient backgrounds dominate the overall background.

For a signal based on measurement of the positron alone, the background is taken to be any event detectable by Cerenkov radiation and depositing $2 \mathrm{MeV}$ or more of energy in the detector. At ranges down to about $10 \mathrm{~km}$, we will see that backgrounds from uranium, thorium, and radon contamination in the water exceed the tolerable background rates just defined. This restriction is relaxed considerably by the addition of a dopant.

\section{Internal Radioactivity}

Uranium and thorium contamination of the water produces $\mathrm{MeV}$ scale beta and gamma rays that are indistinguishable from the positron signal. The uranium and thorium decay chains produce a total of four beta particles and one gamma ray with energies above a 2 $\mathrm{MeV}$ threshold. Table 6 shows the concentration of uranium and thorium required to ensure the rate of positron-like events from internal radioactivity is no greater than the upper limits given in Table 5. For comparison, target uranium and thorium concentrations in the SNO and KamLAND detectors are about $10^{-15}$ to $10^{-16} \mathrm{~g} / \mathrm{g}$. One hundred kilometer and greater range detectors would require several order-of-magnitude reductions in the $\mathrm{U} / \mathrm{Th}$ concentration to bring the internal background below the levels indicated in Table 5. In addition, natural water detectors are ruled out by the severe purity requirements. 
Table 6: The maximum permissible concentration of uranium and thorium in each module operating at the indicated distance.

\begin{tabular}{|l|l|}
\hline $\begin{array}{l}\text { Distance }(\mathrm{km}) \text { of array } \\
\text { from a } 1 \mathrm{kt} \text { explosion }\end{array}$ & $\begin{array}{l}\text { Maximum permissible } \mathrm{U} \text { and Th } \\
\text { concentrations }(\mathrm{g} / \mathrm{g})\end{array}$ \\
\hline 10 & $10^{-16}$ \\
\hline 100 & $9 \times 10^{-19}$ \\
\hline 1000 & $8 \times 10^{-22}$ \\
\hline 12800 & $5 \times 10^{-23}$ \\
\hline
\end{tabular}

${ }^{214} \mathrm{Bi}$ from radon decay also produces a $3.26 \mathrm{MeV}$ beta particle. Although radon is part of the uranium decay chain, it can migrate into the detector from external sources and is treated separately. Table shows the radon concentration per module required to reach the background levels from Table 5.

By sealing the fiducial volume within a low permeability transparent membrane, the KamLAND collaboration expects to achieve a residual radon concentration of $1 \mu \mathrm{Bq} / \mathrm{m}^{3}$ in their active liquid scintillator volume. ${ }^{22}$ Interpolating in Table 7, this concentration in a water detector gives a sensitive range of no more than $40 \mathrm{~km}$.

In summary, at ranges above a few tens of kilometers, the required uranium, thorium and radon concentrations are well below what is currently achievable if only the positron is measured.

Table 7: The maximum permissible concentration of radon in each module operating at the indicated distance.

\begin{tabular}{|l|l|}
\hline $\begin{array}{l}\text { Distance }(\mathrm{km}) \text { of array } \\
\text { from a } 1 \mathrm{kt} \text { explosion }\end{array}$ & $\begin{array}{l}\text { Maximum permissible radon } \\
\text { concentration }\left(\mu \mathrm{Bq} / \mathrm{m}^{3}\right)\end{array}$ \\
\hline 10 & 4.5 \\
\hline 100 & $4 \times 10^{-2}$ \\
\hline 1000 & $4 \times 10^{-5}$ \\
\hline 12800 & $2.5 \times 10^{-6}$ \\
\hline
\end{tabular}

\section{Addition of Dopants to Enhance Sensitivity}

The previous sections demonstrate that radioactive backgrounds would swamp a fission burst signal consisting of 10 events in a 10 second period for undoped water based detectors with ranges greater than a few tens of $\mathrm{km}$. Dopants such as gadolinium, chlorine, or lithium with high neutron absorption cross-sections could dramatically reduce the background rate and allow a relaxation of the severe restrictions on contamination levels. Here we estimate the effects of adding a gadolinium dopant to a light water detector. Gadolinium has the highest neutron capture cross-section of the candidates mentioned, although other materials such as salt are cheaper and more accessible. 


\section{Capture Time, Position Reconstruction and Event Signature}

A 0.1 percent concentration of gadolinium in light water would give a mean neutron capture time of $24 \mu \mathrm{s}$. A $100 \mu$ s time gate between the positron and neutron signal would then give a $94 \%$ neutron capture efficiency. Since the signal neutron travels at most a few tens of centimeters from the antineutrino interaction point prior to capture, the probability of escape from the detector volume is small. After neutron absorption, the excited Gd nucleus decays into $8 \mathrm{MeV}$ of gamma energy, with a typical photon multiplicity of 3 to 4 . While some of these gamma rays are produced below the Cerenkov threshold, a $5 \mathrm{MeV}$ detection threshold should allow recovery of the signal with good efficiency. Liquid scintillator detectors doped at the 0.1 percent level have measured neutron detection efficiencies of about $85-90$ percent with a $6 \mathrm{MeV}$ threshold. ${ }^{23}$

As in liquid scintillator, position reconstruction can be used to define a containment volume for the spatial coincidence between the positron and neutron. A position for each event can be reconstructed using the difference in photon arrival times among struck phototubes. The SuperKamiokande detector, similar in size to the detector considered here, has a vertex resolution of about $0.5 \mathrm{~m}$ in each Cartesian coordinate for $7 \mathrm{MeV}$ electrons, with about 7 struck phototubes per $\mathrm{MeV} .{ }^{24}$ The resolution is fixed by the number of struck phototubes, by the geometry and by the few nanosecond timing resolution of the phototubes.

Figure 7 shows the SuperKamiokande position resolution (black circles) as a function of number of struck phototubes. Extrapolating the Kamiokande vertex resolution function to the lower energies expected here gives a crude estimate of $1 \mathrm{~m}$ resolution in each coordinate for a $2 \mathrm{MeV}$ (25 hit PMT) positron. The higher photostatistics in the present case partially compensates for the lower signal energy.

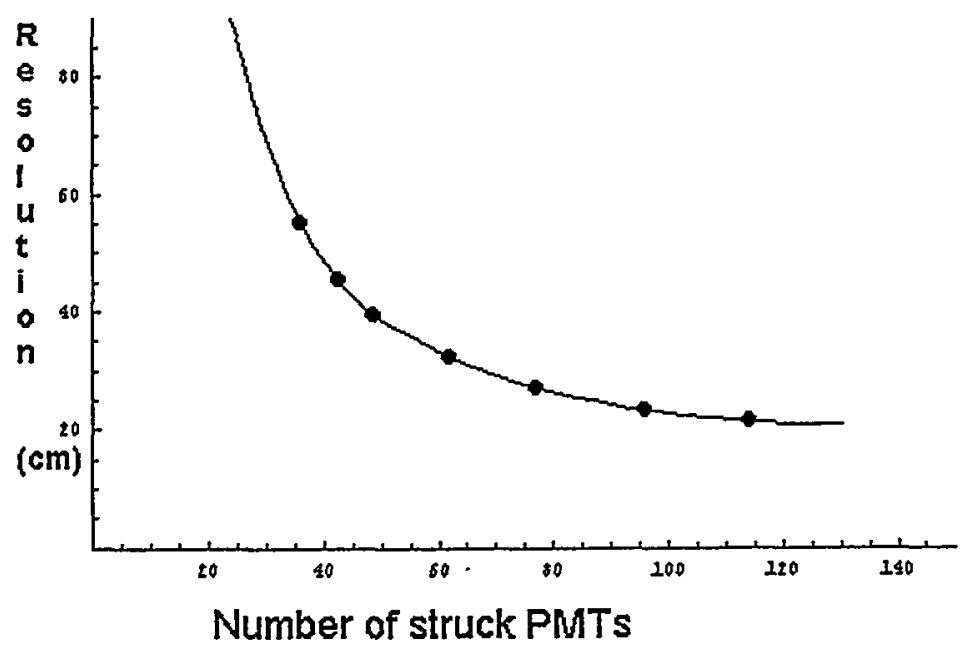

Figure 7: The estimated position resolution extrapolated from SuperKamiokande measurements. 
Using the above capture time and position resolution estimates, a set of cuts can be defined for the coincident antineutrino signal with variables analogous to those in equation 7 . The choice of the reconstructed distance is set by the expected position reconstruction resolution of about $1 \mathrm{~m}$ in each dimension.

$E_{\text {positron }}>2 \mathrm{MeV}$

$\mathrm{E}_{\text {neutron }}>6 \mathrm{MeV}$

$\Delta \mathrm{t} \leq 100 \mu \mathrm{s}$

$|\Delta \mathrm{x}| \leq 4 \mathrm{~m}$

To calculate sensitivity in the presence of a dopant, we require estimates of three backgrounds beyond those for undoped detectors. These are:

- correlated backgrounds from muon-generated fast neutrons that strike proton targets within the detector prior to absorption

- uncorrelated backgrounds produced by the overlap of positron and neutron-like signals within the $100 \mu$ s coincidence gate and the event containment volume

- real antineutrino backgrounds

Positron-like backgrounds from internal radioactivity have been estimated in the previous section. Real antineutrino backgrounds are described in the following section, while the remaining backgrounds are described in Appendix $\Pi$.

\section{Antineutrino Backgrounds}

The most energetic geologic antineutrinos (3.26 MeV) produce positron energies of no more than $1.5 \mathrm{MeV}$ (assuming the positron annihilation gammas are undetected), still below the $2 \mathrm{MeV}$ detection threshold. However, antineutrinos from reactors will ultimately limit the sensitive range of doped detectors, at least with the current design. The rate of reactor antineutrino backgrounds ranges from about 0.5 events per day to several dozen per day per 50,000 ton module depending on location, with the lower extreme obtained in remote locations such as Australia and the South Pole and the upper extreme applicable in regions densely populated with reactors such as France and Japan. 
Table 8: The singles rates from neutron and positron-like coincidental backgrounds in the fiducial volume.

\begin{tabular}{|l|l|l|}
\hline Event Type & & Events/day \\
\hline Neutron-like backgrounds & & $<150,000$ \\
\hline $\begin{array}{l}\text { Muogenic thermal neutrons } \\
\text { at a depth of } 2700 \mathrm{~m} . \mathrm{w} . \mathrm{e} .\end{array}$ & & $<1300$ \\
\hline $\begin{array}{l}\text { Neutron-like signals from } \\
\text { activated oxygen nuclei } \\
(>5 \mathrm{MeV})\end{array}$ & & $<150,000$ \\
\hline $\begin{array}{l}\text { Total neutron-like } \\
\text { background }\end{array}$ & & \\
\hline Positron-like backgrounds & & 6000 \\
\hline $\begin{array}{l}\text { Internal U/TH } \\
\text { Conc. }=\left(10^{-16} \mathrm{~g} / \mathrm{g}\right)\end{array}$ & 6000 \\
\hline $\begin{array}{l}\text { Internal radon } \\
\left(5 \mu \mathrm{Bq} / \mathrm{m}^{3}\right)\end{array}$ & $\begin{array}{l}\text { From PMTs } \\
(0.003 \mathrm{gm} \\
\text { Th)/PMT }\end{array}$ & 12000 \\
\cline { 2 - 3 } $\begin{array}{l}\text { External gammas } \\
\begin{array}{l}\text { (With a } 2.5 \mathrm{~m} \text { water buffer } 1 \mathrm{~m} \text { of rock } \\
\text { surrounding the fiducial } \\
\text { volume })\end{array}\end{array}$ & 13000 \\
\hline $\begin{array}{l}\text { Total positron-like } \\
\text { background } \\
(>2 \mathrm{Mpm} \text { MeV })\end{array}$ & & $\sim 37,000$ \\
\hline
\end{tabular}

\section{Summary of Background Rates}

Table 8 shows the estimated background rates from all uncorrelated positron and neutronlike backgrounds, at the indicated depth and concentrations of contaminants.

The coincidental background rate due to the overlap of uncorrelated neutron and positron like signals is:

13) $r_{\text {acc }}=r_{1} r_{2} \tau \frac{V_{\text {event }}}{V_{\text {fiducial }}}$

with

$$
\begin{array}{ll}
\mathrm{r}_{1}=37,000 \text { per day } & \rightarrow \text { the positron-like singles rate } \\
\mathrm{r}_{2}=150,000 \text { per day } & \rightarrow \text { the neutron-like singles rate } \\
\tau=100 \mu \mathrm{sec} & \rightarrow \text { the PMT time gate } \\
V_{\text {event }} \cong 35 \mathrm{~m}^{3} & \rightarrow \text { the containment volume for the event } \\
V_{\text {fiducial }}=47 \times 10^{3} \mathrm{~m}^{3} & \rightarrow \text { the fiducial volume of the detector }
\end{array}
$$

With these assumptions, the rate of overlap events is about 0.05 per day. If no event position information is used, the rate is about 6 events per day. 
Table 9 shows the background rates in a single module due to reactor antineutrino and correlated non-antineutrino signals.

Table 9: Correlated backgrounds from fast neutrons and from reactor antineutrinos.

\begin{tabular}{|l|l|}
\hline Event type & Events/day \\
\hline Reactor antineutrinos & $\begin{array}{l}\sim 0.5 \text { to several dozen } \\
\text { (depending on detector location) }\end{array}$ \\
\hline $\begin{array}{l}\text { Correlated non- } \\
\text { antineutrino events }\end{array}$ & $\sim 0.3$ \\
\hline
\end{tabular}

The total background rate from all sources is close to 1 event per day per $50,000 \mathrm{~m}^{3}$ module. Referring to Table 5, this gives a limit of around $1000 \mathrm{~km}$ for sensitivity to 10 events produced by a $1 \mathrm{kt}$ test using a doped detector. Even if correlated backgrounds can be further reduced, the reactor background is persistent, allowing extension of the range to only about $1200 \mathrm{~km}$ for a perfectly quiet detector sized to 10 events.

\section{Conclusion}

The above analysis shows that the prospects for detection of antineutrinos from low yield tests are limited, for both on-site and remote applications. Here we summarize the limitations placed on antineutrino-based fission explosion detection at different ranges and in different media.

\section{On-site Liquid Scintillator Detectors}

Within a few kilometers of a test site, possible applications of interest are:

- cooperative monitoring of a restricted region of an existing nuclear test site for $1 \mathrm{kt}$ explosions

- cooperative monitoring of a sensitive area designated by the host country to reduce the likelihood of CTBT on-site verification inspections occuring within this "inspection exclusion" zone

- verifying that sub-critical tests are not in fact low yield super-critical explosions nor being used to mask such explosions

Existing liquid scintillator detectors constructed about 50 m.w.e. (20 m of rock) below the surface could detect $1 \mathrm{kt}$ tests a few kilometers from a source and therefore could be useful for the first two applications. The utility of such a detector ultimately depends on trade-offs between cost and the desire by states for a non-intrusive local nuclear monitoring system.

Going down to yields in the few kilogram range for monitoring sub-critical tests is much more difficult. For example, an array of about 3050,000 ton doped water modules (i.e., 
30 SuperKamiokande sized detectors) would be required to detect 10 events from a 5 kilogram yield test $100 \mathrm{~m}$ away from the test location. Such an application might be of interest only if a detector array were built for other purposes, such as physics research or for longer range monitoring of higher yield tests.

\section{Water Detectors}

Highly pure water detectors without dopants are not feasible at ranges greater than a few tens of kilometers because of the overwhelming backgrounds from ambient radioactivity. Dopants such as gadolinium or chlorine that allow for neutron recovery could help reduce the background rate dramatically. Gadolinium is too expensive $(\sim 100$ per gram) to be feasible for large arrays, but detectors using chlorine salts would be less expensive. As an example, the SNO detector in Canada has the capability of cycling 2.5 tons of salt through a 1000 ton active medium (heavy water).

If dopants can be used, detection of $1 \mathrm{kt}$ tests at the $100 \mathrm{~km}$ range up to about $1000 \mathrm{~km}$ is limited primarily by three engineering obstacles. First, the cost of photomultiplier tubes is now around several hundred to a few thousand dollars per tube, while the number of tubes required for remote detection ranges from several hundred thousand to several billion or more. This obstacle is clearly insuperable without dramatic decreases in the cost of photodetection. Collection and detection efficiencies of the phototubes, set in our study to 50 percent and 20 percent respectively, may increase by no more than an overall factor of two or three at most, with a similar decrease in cost. The second engineeringrelated obstacle is the feasibility and cost of purifying water on the large scale required for detection at great distances. Target purification levels of $10^{-15}$ to $10^{-16} \mathrm{~g} / \mathrm{g}$ are within the reach of current technology, but the amount of water treated would have to be scaled up by at least two orders of magnitude for remote applications. The third obstacle is the requirement of deep burial of the detector to screen out cosmic backgrounds. At a sensitive range of around $1000 \mathrm{~km}, 6300$ modules would have to be built about $1100 \mathrm{~m}$ below ground to achieve sufficient screening of cosmic rays. The only large-scale projects approaching this size and depth are nuclear waste repositories. For example, the Yucca Mountain repository will consist of several miles of tunnels 5 to $10 \mathrm{~m}$ in diameter constructed at a depth of 200 to $300 \mathrm{~m}$ below ground.

Most of the backgrounds discussed in the previous section can be minimized, at least in principle, by shielding, purification or other engineering steps. However, beyond approximately $1000 \mathrm{~km}$ there is a physics limitation imposed by the real antineutrino background from the world's fission reactors. Even assuming the complete absence of non-antineutrino backgrounds (i.e., a perfectly quiet detector) the rate in a single 50,000 ton module from this source is about 0.5 . events per day in the world's most remote regions, which, interpolating in Table 5, gives a range of no more than $1200 \mathrm{~km}$ for a $1 \mathrm{kt}$ explosion. This limit can be evaded by requiring more than 10 signal events. However, this would require detector arrays even larger than those contemplated here, with the array size rising faster than the square of the test-to-detector distance above $1000 \mathrm{~km}$. 
Taking all of these factors into account, the greatest range that seems possible in the next decade or so is a distance of $100 \mathrm{~km}$ from a $1 \mathrm{kt}$ test, requiring about 6050,000 ton water modules. This is 60 times larger than the largest neutrino detector now in operation. The $100 \mathrm{~km}$ range is sufficient to cover existing test sites in nuclear weapons states, such as the Nevada Test Site and Semipalatinsk sites.

In short, while antineutrino detectors are in theory very attractive for CTBT verification, both engineering difficulties and physics limitations severely limit the actual application of the technology for this purpose. Nuclear test monitoring applications which are clearly feasible using existing antineutrino detectors are probably of only very limited utility, while applications of greater utility, at greater distances, are not feasible without significant advances in detection technology beyond the current state of the art. Table 10 summarizes these findings.

Table 10: The applications and feasibility of antineutrino detection at various ranges from a $1 \mathrm{kt}$ fission explosion.

\begin{tabular}{|c|c|c|}
\hline $\begin{array}{c}\text { Range } \\
\text { (1 kt, } 10 \text { events) }\end{array}$ & Applications & Feasibility \\
\hline $10 \mathrm{~km}$ & $\begin{array}{l}\text { Cooperative monitoring of few } \\
\text { sq } \mathrm{km} \text { areas of former test sites } \\
\text { Cooperative monitoring to create } \\
\text { CTBT on-site inspection } \\
\text { "exclusion zones" }\end{array}$ & $\begin{array}{l}\text { Possible with current liquid } \\
\text { scintillator detectors built at } \\
\text { depths of } 50 \text { m.w.e. }\end{array}$ \\
\hline $100 \mathrm{~km}$ & $\begin{array}{l}\text { Detection of } 1 \mathrm{kt} \text { tests over } \\
\text { entire test sites } \\
\text { Cooperative monitoring of } \\
\text { subcritical tests placed near the } \\
\text { detector array } \\
\text { See over borders for larger tests }\end{array}$ & $\begin{array}{l}\text { At limits of current technology } \\
\text { - Must recover neutron } \\
\text { signal through doping } \\
\text { - Cheaper photodetection } \\
\text { would help (current } \\
\text { phototube noise } \\
\text { acceptable) }\end{array}$ \\
\hline $1000 \mathrm{~km}$ & $\begin{array}{l}\text { See over borders } \\
\text { Cover entire countries/regions of } \\
\text { interest }\end{array}$ & $\begin{array}{l}\text { Significant advances needed } \\
\text { beyond current technology } \\
\text { - Lower cost photodetection } \\
\text { - Improvements in large } \\
\text { scale water purification } \\
\text { - Recovery of neutron signal } \\
\text { - New detection } \\
\text { methods/ways to reduce } \\
\text { background }\end{array}$ \\
\hline$>1000 \mathrm{~km}$ & Remote monitoring of $1 \mathrm{kt}$ tests & $\begin{array}{l}\text { Impossible for this design type } \\
\text { and number of events due to } \\
\text { irreducible reactor } \\
\text { backgrounds }\end{array}$ \\
\hline
\end{tabular}




\section{Appendix l: Intrinsic Phototube Noise}

Intrinsic phototube noise, or dark current, arises from spontaneous ejection of electrons from the photocathode surface due to thermal fluctuations. Rates range from one hundred to several thousand single photoelectron events per second per phototube. Because of the weak signal and absence of any time coincidence dark current can be a significant background to the antineutrino pulse in undoped $\mathrm{H}_{2} \mathrm{O}$ detectors. Here we show that existing phototubes are quiet enough to allow for the use of modules of the size prescribed above.

We define a noise equivalent event as the triggering of 25 or more phototubes in $200 \mathrm{~ns}$ by phototube dark current, corresponding to the $2 \mathrm{MeV}$ threshold for event selection. The rate of such events in each module is given by:

$$
\mathrm{R}_{\mathrm{acc}}^{\mathrm{n}}=\sum_{\mathrm{n}=\mathrm{N}_{\mathrm{pe}}}^{\mathrm{N}_{\mathrm{PMT}}} \frac{\mathrm{N}_{\mathrm{PMT}} !}{(\mathrm{n} !)\left(\mathrm{N}_{\mathrm{PMT}}-\mathrm{n}\right) !} \times \mathrm{n} \times(\lambda)^{\mathrm{n}}\left(\tau_{\mathrm{PMT}}\right)^{\mathrm{n}-1} ; \lambda \tau \ll 1
$$

$\lambda$

$\rightarrow$ the background rate per tube

$\tau_{\mathrm{PMT}}=200 \mathrm{~ns} \rightarrow$ the time gate

$\mathrm{N}_{\mathrm{PMT}}=16200 \rightarrow$ the number of tubes in a single module

$\mathrm{n} \quad \rightarrow$ the number of struck phototubes

$N_{\mathrm{pe}} \quad \geq 25(2 \mathrm{MeV}) \rightarrow$ the minimum number of photoelectrons required for a trigger

This series (which is dominated by its first few terms) can be solved for $\lambda$ to give a maximum acceptable dark current rate per tube. The rate decreases with detector range since the overall acceptable noise rate per module decreases. Table 11 shows the maximum allowable phototube dark current versus range required to ensure a PMT noise level lower than the overall noise rate given in Table 5 . These rates are slightly lower than those in existing large phototubes. For example, the actual dark current rate in 50.8 $\mathrm{cm}$ tubes used in the SuperKamiokande experiment is about $3 \mathrm{kHz}$. ${ }^{25}$ Thus, phototubes with intrinsic photostatistical noise levels comparable to the present state of the art can be used even in fairly long-range detectors.

Table 11: The dark current level per phototube required to ensure that phototube noise is no greater than the overall tolerable noise level per module.

\begin{tabular}{|c|c|}
\hline Range (km) & Maximum allowable dark current $(\mathbf{H z})$ \\
\hline 10 & 1300 \\
\hline 100 & 1100 \\
\hline 1000 & 950 \\
\hline 12,800 & 750 \\
\hline
\end{tabular}




\section{Appendix II: Backgrounds In Doped Water Detectors}

\section{Correlated Backgrounds}

Muons with energies in the $10-100 \mathrm{GeV}$ range passing through the fiducial volume can deposit several $\mathrm{GeV}$ of energy by ionization. These events are easily rejected by making a maximum energy cut. Fast spallation neutrons produced by these muons can also be rejected by a muon veto.

High energy, muon-generated, spallation and capture neutrons, with energies of $10 \mathrm{MeV}$ up to about $1000 \mathrm{MeV}$, are generated in the rock surrounding the detector. These can enter the detector volume without being vetoed, strike proton targets in the water to produce a positron-like signal, and then thermalize and capture on the dopant within a $100 \mu$ s time window. We derive an approximate upper bound on this rate by scaling from Monte-Carlo estimates made by the KamLAND collaboration for neutrons produced in rock at a depth of 2700 m.w.e. ${ }^{26}$ The KamLAND estimates are based on a $1 \mathrm{MeV}$ energy threshold for the positron-like signal, a 1.8 - 2.7 MeV energy range for the neutron-like signal, a 10 to $500 \mu$ s time coincidence gate, and a positron-neutron separation distance of no more than $1 \mathrm{~m}$. These criteria are all less strict than those used for the detector considered here except for the stricter proximity requirement for the positron and neutron signals. However, this requirement will have little effect on the rate. Neutrons elastically scattering from proton targets transfer one half of their energy on average to the proton. Therefore fast neutrons that survive the initial $1 \mathrm{MeV}$ threshold cut will have energies of no more than a few $\mathrm{MeV}$ and will travel no more than a few tens of centimeters from their initial interaction point before thermalization. For this reason, the KamLAND estimates can be used to provide an upper bound on the event rate in the detector considered here.

For a spherical shell $1 \mathrm{~m}$ in thickness with a $50 \mathrm{~m}$ inner diameter (to account for a $2.5 \mathrm{~m}$ thick water buffer) the scaled neutron production rate is about 56,000 events/day. After attenuation through the buffer, no more than 13 neutrons per day with energies greater than $1 \mathrm{MeV}$ will enter the $45 \mathrm{~m}$ diameter fiducial volume. The rate of correlated events using the KamLAND selection criteria is about 0.3 per day. The event rate in a water detector from these neutrons will be smaller than this due to the stricter energy and time coincidence requirements. 
Table 12: The approximate background rate from spallation neutrons produced in rock surrounding the detector.

\begin{tabular}{|l|l|l|}
\hline $\begin{array}{l}\text { Spallation events } \\
\text { generated in } 1 \mathrm{~m} \\
\text { thickness of rock } \\
\text { surrounding a } 50 \mathrm{~m} \\
\text { diameter volume }\end{array}$ & $\begin{array}{l}\text { Uncorrelated events } \\
\text { surviving in the fiducial } \\
\text { volume (neutrons } \\
\text { depositing }>1 \mathrm{MeV} \text { of } \\
\text { energy) }\end{array}$ & $\begin{array}{l}\text { Correlated events in the } \\
\text { fiducial volume }\end{array}$ \\
\hline $56,000 /$ day & $<13 /$ day & $<0.3 /$ day \\
\hline
\end{tabular}

\section{Uncorrelated Backgrounds}

To estimate the uncorrelated background, we require the total rate of uncorrelated positron and neutron-like signals within the detector volume. Below we estimate the positron-like backgrounds from external sources and the neutron singles rate. (The rate of positron-like backgrounds from internal sources has already been estimated in the main body of the article.)

\section{Positron-like Events From External Radioactivity}

External radioactivity comes from uranium and thorium in support structures, photomultiplier tubes, and external rock, with the latter two sources dominating the rate. Beta particles will be screened out by the thick water shield around the fiducial volume. However, there is a $2.62 \mathrm{MeV}$ penetrating gamma from the thorium decay chain. ${ }^{27}$ This background can be reduced by the use of a graded shield consisting of the water shield and relatively low activity rock or gravel. To be specific, we assume a $2.5 \mathrm{~m}$ thick water buffer, followed by the phototube array with $0.03 \mathrm{~g}$ of uranium and thorium per tube, followed by a $1 \mathrm{~m}$ thick region of rock with U/TH concentrations of $1 \mathrm{ppm}$. The background rate due to the rock saturates beyond about $1 \mathrm{~m}$.

The phototubes are approximated as a smooth spherical shell of radioactive material 2.5 $\mathrm{m}$ from the fiducial volume. Contributions from surrounding rock are built up by summing the thin shells out to $1 \mathrm{~m}$. To obtain a rate, a differential flux through a patch on the fiducial surface is obtained by numerically integrating the quantity:

$$
\frac{e^{-\mu x}}{4 \pi x^{2}}
$$

over the outer shell, with $\mu$ the attenuation length for $3 \mathrm{MeV}$ photons. Figure 8 shows the geometry and the definition of the integration variable $\mathrm{x} . \mathrm{x}$ extends over the range:

$$
\mathrm{t}<\mathrm{x}<\sqrt{\mathrm{r}^{2}-\mathrm{r}_{\mathrm{m}}^{2}}
$$

with $t$ the buffer thickness, $r_{m}$ the fiducial volume and $r$ the radius at which the phototubes or rock shells are located, as shown in Figure 8. 
The total rate from each shell is the integral of this flux over the inner surface.

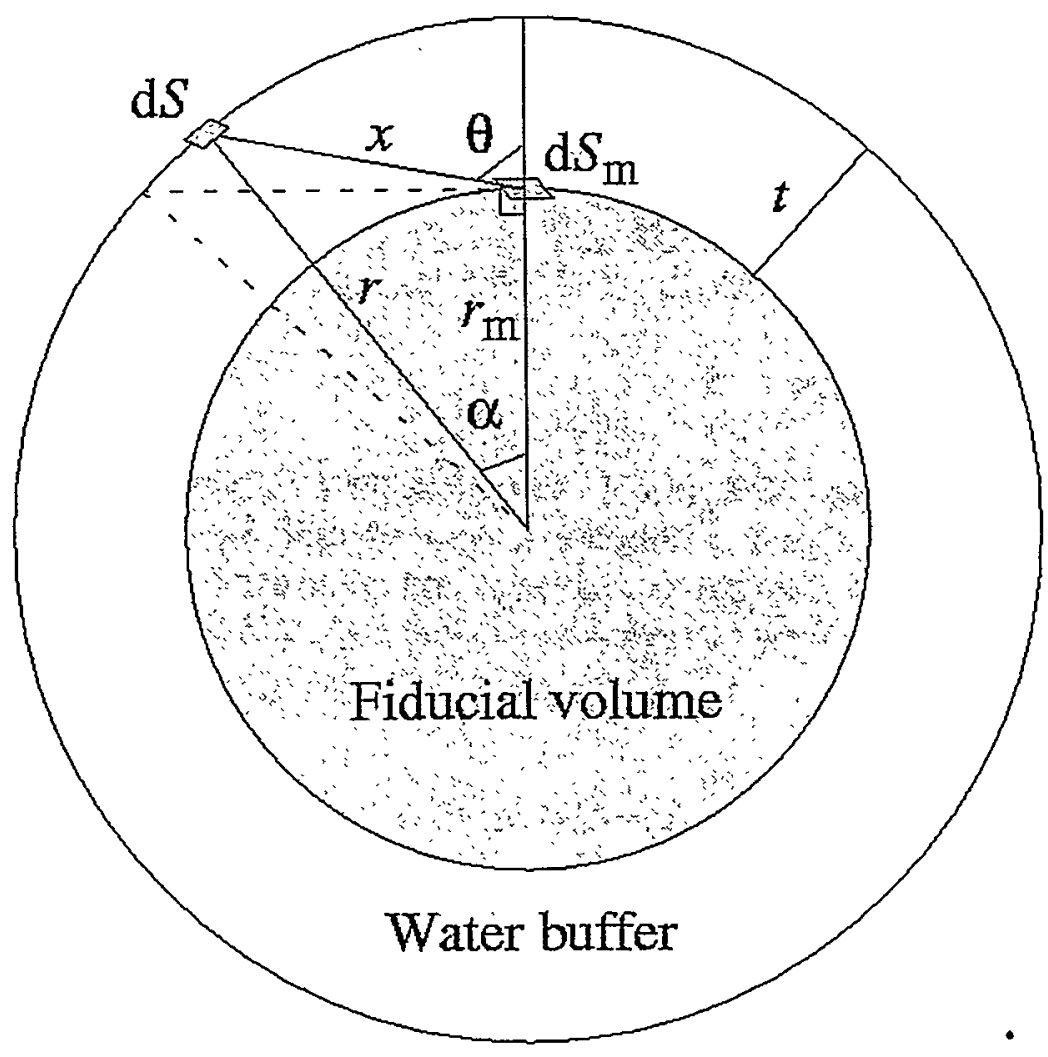

Figure 8: The variables used to calculate the background rate in the fiducial volume from PMT and external rock radioactivity.

The setback of the phototubes in this case would require an increase in the number of phototubes per module to maintain photostatistics. This increase is shown along with the background rates from both sources in Table 13.

Table 13: The positron-like background rates from external sources and the increase in the number of phototubes required to maintain equivalent photostatistics for a water buffer of the indicated thickness. The number of tubes without a buffer is 16,200 (45 m diameter module).

\begin{tabular}{|c|c|c|c|}
\hline Buffer Thickness (m) & \multicolumn{2}{|c|}{$\begin{array}{c}\text { Background rate } \\
\text { (events/day) } \\
\text { rock }\end{array}$} & $\begin{array}{c}\text { Number of } \\
\text { phototubes per } \\
\text { module }\end{array}$ \\
\hline 2.5 & 12,000 & 13,000 & 20,000 \\
\hline
\end{tabular}




\section{Neutron-Like Backgrounds}

Neutron-like signals come from actual neutrons produced and captured within the detector by muons, as well as equivalent signals from $>6 \mathrm{MeV}$ gamma or beta particles produced by activation of nuclei with widely varying decay times. For the latter rate, a study of spallation products by the SuperKamiokande collaboration predicts about 1300 events per day above a $6.5 \mathrm{MeV}$ threshold scaled to the 50,000 ton fiducial volume. ${ }^{28}$

The thermal neutron production rate within the detector is difficult to estimate accurately. However, our previous estimate of the rate of muon generated neutron production in rock can be scaled to give a conservative upper bound on the rate of thermal neutron production in water. The previous estimate assumes a rock density of $2.7 \mathrm{gm} / \mathrm{cm}^{3}$. Scaling the rate to water and assuming that all neutrons produced by muons within the fiducial volume of the detector thermalize, capture on gadolinium, and escape a muon veto, we obtain an upper bound on the uncorrelated rate of 147,000 neutrons per day in the fiducial volume. Of course, this rate is greatly reduced by the requirement of spatial and time coincidence with the positron signal.

\section{Acknowledgements}

We gratefully acknowledge the support for this work provided by the CTBT Verification Research and Development Department and the International Security Center at Sandia National Laboratories, Albuquerque, and the Center for Exploratory Systems and Development at Sandia National Laboratories, California.

We wish to thank Professor Buford Price and Dr. Kurt Woschnagg of the Physics Department at University of California, Berkeley for their valuable assistance in describing and characterizing the properties of the AMANDA detector and other large scale antineutrino detectors.

\footnotetext{
${ }^{1}$ http://www.ctbt.rnd.doe.gov/ctbt/introduction/onsite_inspections.html.

${ }^{2}$ See for example, T. Bowles and M. Silbar, "the Neutrino in 1980", Los Alamos Science, Winter/Spring 1981, Vol. 2, No. 1, p. 94.

${ }^{3}$ Information about SuperKamiokande can be found at http://www-sk.icrr.u-tokyo.ac.jp/doc/sk/superkamiokande.html, while the cubic kilometer ice detector is described at http://amanda.berkeley.edu/km3. ${ }^{4}$ Given this technical limitation in the current international monitoring system, on-site inspections (OSI) will be relied upon to gather evidence on ambiguous or suspect events.

${ }^{5}$ The third false positive occurred in Pakistan on April 28, 1991. Pakistani scientists incorrectly suspected that the event was an Indian nuclear test. Gregory van der Vink and Terry Wallace, "The Political Sensitivity of Earthquake Locations," IRIS Newsletter, Vol. 15, No. 3, Fall 1996, pp. 20-23. Gregory van der Vink and Terry Wallace, "Response to Comments by M. Henger, K. Koch, B. Ruud, and E. Husebye," IRIS Newsletter, vol. 16, no. 2, Fall/Winter 1997, pp. 21-22.

${ }^{6}$ Seismologists have incorrectly classified nuclear explosive tests as earthquakes before. See S. Arora and T. Basu, "A Source Discrimination Study of a Chinese Seismic Event of May 4, 1983," Tectonophysics, no. 109,1984, pp. 241-251.

${ }^{7}$ W.J. Hannon, "Seismic Verification of a Comprehensive Test Ban," Science, Vol. 227, No. 4684, January 18,1985, pp. 251-257.
} 
${ }^{8} \mathrm{http}: / /$ www-sk.icrr.u-tokyo.ac.jp/doc/sk/super-kamiokande.html, http://amanda.berkeley.edu/km3, op. cit.

${ }^{9} \mathrm{http} / / / \mathrm{www} . n v . d o e . g o v / n e w s \% 26$ pubs/newsreleases/archives/pr970702.htm.

${ }^{10}$ Pulsed reactors could produce weak antineutrino bursts with a variety of time scales.

${ }^{11}$ Other detection methods, which rely on radiochemical conversion of nuclei by neutrinos, have cycle times that are too slow to be useful for detecting fission explosions in real time.

${ }^{12}$ Gadolinium has a neutron capture cross section of about 49,000 barns, compared with 0.328 barns for hydrogen.

${ }^{13}$ Detector parameters are taken from F. Suekane, "Status of the KamLAND Experiment", Talk presented at Europhysics Neutrino Oscillation Workshop, (Now'98) 7-9 September 1998, Amsterdam, the Netherlands.

${ }^{14} \mathrm{http} / / / \mathrm{www}$. Ings.infn.it/lngs/htexts/lvd/.

${ }^{15} \mathrm{~F}$. Suekane, ICRC talk July 98.

${ }^{16}$ Y. Totsuka, "Experiments for Solar Neutrinos and Supernova Neutrinos," in Physics and Astrophysics of Neutrinos, M Fukugita, A. Suzuki, eds., (Springer Verlag: Tokyo, 1994) p. 687

${ }^{17}$ R.S. Raghavan, S. Schoenert, S. Enomoto, J. Shirai, F. Suekane, A. Suzuki, "Measuring the Global Radioactivity in the Earth by Multidetector Antineutrino Spectroscopy," Phys. Rev. Lett. Vol. 80, Number 3 , p. 635, (1998).

${ }^{18}$ C. Rothschild, M. Chen, F. Calaprice, "Antineutrino Geophysics with Liquid Scintillator Detectors," http://xxx.lanl.gov/abs/nucl-ex/9710001, Submitted to Geophysical Research Letters, 15 Oct. 1997.

${ }_{19}^{19}$ M. Aglietta et al., "Neutron Flux Generated by Cosmic-Ray Muons at $5200 \mathrm{hg} / \mathrm{cm}^{2}$ s.r. Underground. Depth Intensity Curve," Il Nuovo Cimento, Vol. 12 C. N. 4, July-August 1989, p. 467.

${ }^{20}$ H.H. Chen, "The Sudbury Neutrino Observatory," Nuclear Instruments and Methods in Physics Research A264, p. 50. (1988).

${ }^{21}$ Cerenkov light is a coherent electromagnetic shock wave emitted when a charged particle travels through a medium at speeds greater than the speed of light in the medium.

${ }^{22}$ P. Alivisatos, et al., "KamLAND, a Liquid Scintillator Anti-Neutrino Detector at the Kamioka Site," Preprint Stanford-HEP-98-03; Tohoku-RCNS-98-15 (Geneva:Cern Library SCAN-9809050), at http://alice.cern.ch/search/complex?uid=3470113_2649\&freetext1=Alivisatos+P\&field1=wau, p. 60.

${ }^{23} \mathrm{H}$. de Kerret et al., "Proposal to Search for Neutrino Vacuum Oscillations Using a $1 \mathrm{Km}$ Baseline Reactor Neutrino Experiment," 1993, http://www.hep.anl.gov/NDK/Hypertext/chooz.html, p. 74.

${ }^{24}$ T. Yamaguchi, Study of Solar Neutrinos at Super-Kamiokande, Thesis, University of Tokyo, April. 1988, http://www-sk.icrr.u-tokyo.ac.jp/doc/sk/pub/index.html, p.75.

${ }^{25}$ Ibid., p. 37.

${ }^{26}$ P. Alivisatos et al., op. cit., p. 52.

${ }^{27}$ No other decay in the U, Th (or K) decay chains has gamma energies greater than $2 \mathrm{MeV}$.

${ }^{28}$ T. Yamaguchi, op. cit., p. 77. 


\section{DISTRIBUTION:}

1 MS 0151 Gerold Yonas, 16000

1 MS 0979 Larry Walker, 5704

1 MS 1202 Horace Poteet, 5933

1 MS 1207 Dave Waymire, 5914

1 MS 1211 Chuck Carson, 5312

1 MS 1215 Rich Preston, 5336

1 MS 1219 Charles Craft, 5941

1. MS 9001 Mim John, 8000

1 MS 9004 John Vitko, 8100

1 MS 9103 Greg Thomas, 8120

1 MS 9201 Larry Brandt, 8112

1 MS 9201 Vipin Gupta, 16000

1 MS 9201 Pat Falcone, 8114

1 MS 9201 Todd West, 8114

15 MS 9214 Adam Bernstein, 8112

3 MS 9018 Central Technical Files, 8940-2

1 MS 0899 Technical Library, 4916

1 MS 9021 Technical Communications Department, 8815/

Technical Library, MS 0899, 4916

1 MS 9021 Technical Communications Department, 8815 For DOE/OSTI 
This page intentionally left blank 